\title{
Targeting VLA4 integrin and CXCR2 mobilizes serially repopulating hematopoietic stem cells
}

\author{
Darja Karpova, ${ }^{1,2}$ Michael P. Rettig, Julie Ritchey, ${ }^{1}$ Daniel Cancilla, ${ }^{1}$ Stephanie Christ, ${ }^{1}$ Leah Gehrs, ${ }^{1}$ Ezhilarasi Chendamarai, ${ }^{1}$ \\ Moses O. Evbuomwan, ${ }^{3}$ Matthew Holt, ${ }^{1}$ Jingzhu Zhang, ${ }^{1}$ Grazia Abou-Ezzi, ${ }^{1}$ Hamza Celik, ${ }^{1}$ Eliza Wiercinska, ${ }^{4}$ Wei Yang, ${ }^{5}$ Feng Gao, ${ }^{6}$ \\ Linda C. Eissenberg, ${ }^{1}$ Richard F. Heier, ${ }^{7}$ Stacy D. Arnett, ${ }^{7}$ Marvin J. Meyers, ${ }^{7}$ Michael J. Prinsen, ${ }^{7}$ David W. Griggs, ${ }^{7}$ Andreas Trumpp, ${ }^{2}$ \\ Peter G. Ruminski, ${ }^{1,7}$ Dwight M. Morrow, ${ }^{8}$ Halvard B. Bonig, ${ }^{4,9}$ Daniel C. Link, ${ }^{1}$ and John F. DiPersio ${ }^{1}$ \\ 'Division of Oncology, Department of Medicine, Washington University School of Medicine, St. Louis, Missouri, USA. 'Division of Stem Cells and Cancer, German Cancer Research Center (DKFZ) and DKFZ- \\ ZMBH Alliance, Heidelberg, Germany. ${ }^{3}$ Oakland University William Beaumont School of Medicine, Rochester, Michigan, USA. ${ }^{4}$ Cerman Red Cross Blood Service and Institute for Transfusion Medicine and \\ Immunohematology of the Goethe University, Frankfurt, Germany. ${ }^{5}$ Cenome Technology Access Center, Washington University, St. Louis, Missouri, USA. ${ }^{6}$ Division of Public Health Sciences, Department \\ of Surgery, Washington University School of Medicine, St. Louis, Missouri, USA. 'enter for World Health and Medicine, Saint Louis University, St. Louis, Missouri, USA. ${ }^{8}$ Magenta Therapeutics, Cambridge, \\ Massachusetts, USA. ' University of Washington, Department of Medicine/Hematology, Seattle, Washington, USA.
}

\begin{abstract}
Mobilized peripheral blood has become the primary source of hematopoietic stem and progenitor cells (HSPCs) for stem cell transplantation, with a 5-day course of granulocyte colony-stimulating factor (G-CSF) as the most common regimen used for HSPC mobilization. The CXCR4 inhibitor plerixafor is a more rapid mobilizer, yet not potent enough when used as a single agent, thus emphasizing the need for faster acting agents with more predictable mobilization responses and fewer side effects. We sought to improve hematopoietic stem cell transplantation by developing a new mobilization strategy in mice through combined targeting of the chemokine receptor CXCR2 and the very late antigen 4 (VLA4) integrin. Rapid and synergistic mobilization of HSPCs along with an enhanced recruitment of true HSCs was achieved when a CXCR2 agonist was coadministered in conjunction with a VLA4 inhibitor. Mechanistic studies revealed involvement of CXCR2 expressed on BM stroma in addition to stimulation of the receptor on granulocytes in the regulation of HSPC localization and egress. Given the rapid kinetics and potency of HSPC mobilization achieved by the VLA4 inhibitor and CXCR2 agonist combination in mice compared with currently approved HSPC mobilization methods, the combination represents an exciting potential strategy for clinical development in the future.
\end{abstract}

Authorship note: DK and MR contributed equally to this work. Conflict of interest: DK and JFD have a patent application (PCT/US2017/059770, compositions comprising integrin inhibitors and agents that interact with a chemokine receptor). PGR, MJM, RFH, MPR, and JFD have pending patent applications (PCT/US2017/059777, integrin inhibitors and chemokine receptor agents; PCT) US2017/059733, integrin antagonists) and report royalties received from the patent applications during the conduct of the study. PGR, MJM, RFH, MPR, DWG, and JFD have one or more additional patents listed in an appendix in the supplement. JFD has a first-tier potential conflict due to equity ownership in Magenta Therapeutics Inc. and WUGEN Inc. JFD is founder and advisor for Magenta Therapeutics Inc. and WUGEN Inc He receives income from RiverVest Venture Partners and Magenta Therapeutics Inc.; he receives research funding from Amphivena Therapeutics, NeoimmuneTech Inc., Mac rogenics Inc., Incyte Corp., BiolineRx, Altiris, and WUGEN Inc.; he is an Advisory Board Member for Cellworks Group, RiverVest Venture Partners, and Arch Oncology. DMM is an employee of and owns equity in Magenta Therapeutics Inc. PGR has stock in Pfizer Inc. PGR and DWG are cofounders of, consultants for, and own equity in Indalo Therapeutics, a company pursuing clinical development of RCD-binding integrin antagonists, but not of antagonists of a $\alpha 4 \beta 1$ (VLA4). MPR serves as a consultant for RiverVest Venture Partners, and has received research funding from Amphivena Therapeutics, Novimmmune, and Cantex. MJM receives research funding from Ultragenyx Pharmaceutical Inc. and is the founder and owner of Meyers MedChem Consulting LLC. RFH is currently an employee of Confluence Discovery Technologies. LE has received research funding from MaxCyte Inc. HBB is a coinventor of patent PCT/EP2015/066083 from which he receives royalties and licensing fees; he has received honoraria from Miltenyi, Celgene, Sandoz-Hexal, Novartis, and Terumo BCT; he has received research support from Sandoz-Hexal, Amgen, Polyphor, Stage (now Juno), Miltenyi, Terumo BCT; and he has served on the advisory boards of Genzyme, Novartis, Celgene, and Terumo BCT. Copyright: (c) 2019, American Society for Clinical Investigation.

Submitted: September 6, 2018; Accepted: April 25, 2019; Published: June 10, 2019 Reference information: J Clin Invest. 2019;129(7): 2745-2759. https://doi.org/10.1172/JCl124738.

\section{Introduction}

In postnatal mammals, the vast majority of hematopoietic stem and progenitor cells (HSPCs) reside in the protective environment of the bone marrow $(\mathrm{BM})(1,2)$. Accordingly, only very few HSPCs can be found in the periphery, primarily in blood and spleen, during homeostasis (3). A wide variety of stimuli has been identified that elicits HSPC egress from the marrow, a phenomenon referred to as mobilization (4). Despite its significance and the preclinical discovery of various approaches to lure HSPCs into the circulation during the past 2 decades, the armamentarium of clinical mobilization remains sparse. Thus, granulocyte colony-stimulating factor (G-CSF) is the sole agent approved for mobilization of healthy donors, whereas in patients chemotherapy and the small molecule antagonist of the chemokine receptor CXCR4, AMD3100 (Plerixafor), are approved in conjunction with G-CSF (5).

Limitations of currently available clinical regimens provide the rationale for the ongoing search for alternative mobilization strategies. Importantly, all approaches described to date target at least 1 of the 2 major axes mediating HSPC retention: the chemokine receptor CXCR4 or the integrin very late antigen 4 (VLA4) $(4,6,7)$. Inhibition of the interaction between CXCR4 and its chief ligand CXCL12 has been extensively studied by us and others (8-13). By contrast, targeting of VLA4 and use thereof to release HSPCs from the BM has been explored considerably less intensively due to lack of suitable small molecule compounds with favorable pharmacologic properties $(7,14)$. 
The chemokine receptor CXCR2 is a critical regulator of neutrophil chemotaxis (15). Interestingly, stimulation of CXCR2 signaling (e.g., with its ligands CXCL1, -2 , or -8 ) has been demonstrated to result in rapid HSPC mobilization from the BM even though the receptor is not expressed on HSPCs themselves (1620). The mechanism of HSPC egress after CXCR2 stimulation remains controversial.

In this study, we explored the potential of combined targeting of VLA4 and CXCR2 signaling as a novel strategy to mobilize HSPCs. In addition to being associated with a rapid and remarkably synergistic HSPC release, administration of a VLA4 antagonist in conjunction with a CXCR2 ligand targeted very primitive, serially repopulating HSPCs with high efficiency. Our observations challenge the notion of limited potential of fast-acting mobilizing agents and are particularly relevant in the context of the ongoing debate about the vascular versus endosteal localization of stem cells (21-23). Moreover, unexpectedly, stromal cells were found to be targeted by CXCR2 stimulation along with neutrophils. This contribution of both stromal and neutrophilic CXCR2 to the regulation of HSPC localization underlies the intricate interplay between nonhematopoietic and mature hematopoietic cells for HSPC maintenance.

\section{Results}

Combined targeting of VLA4 and CXCR 2 results in augmented HSPC recruitment. We assessed the mobilization efficiency of a VLA4 antagonist alone compared with a CXCR2 agonist alone, as well as their combined effect in vivo. The previously described smallmolecule VLA4 inhibitor firategrast $(24,25)$ along with the naturally occurring truncated form of the CXCR2 ligand Gro- $\beta$ (tGro- $\beta$ ) $(26,27)$ were used in the initial experiment. As shown in Figure 1, combined treatment with both agents resulted in increased numbers of circulating WBCs and CFU-Cs of up to 3- and 10-fold, respectively (Figure 1, A and B). To circumvent the shortcomings of VLA4-targeting compounds hitherto tested as mobilizing agents, we selected a series of VLA4 antagonists that had been developed based on the structure of the well-known VLA4 inhibitors BIO5192 (28) and firategrast $(24,25)$. These compounds (CWHM-822, $823,824,825$, and 842; Figure 1C) were synthesized as previously described (29-34). Properties of the inhibitors were assessed using a colorimetric, cell-free, solid-phase receptor binding assay (SPRA; Supplemental Table 1; supplemental material available online with this article; https://oi.org/10.1172/JCI124738DS1) as well as a flow cytometry-based soluble VCAM1 binding assay (Figure 1D). CWHM-823, -824, and -842 showed superior binding affinity to VLA4 compared with firategrast (Figure 1D). CWHM-822 and -823 had an improved water solubility compared with both BIO5192 and firategrast (Supplemental Table 1).

We next tested whether the synergism between VLA4 inhibition and CXCR2 stimulation was a compound class as opposed to a compound-specific effect. Therefore, mobilization with BIO5192 and firategrast was tested alongside the new compounds, CWHM-823 and -842. All 4 inhibitors mobilized HPSCs by themselves, whereas the mobilization response was enhanced up to 3 - to 10 -fold when combined with tGro- $\beta$ (Figure 1E), suggesting a compound class-specific effect. Firategrast-related CWHM-823 outperformed the BIO5192-related CWHM-842 in vivo and was therefore selected for the majority of our subsequent analyses.
Optimal pharmacokinetics and pharmacodynamics were determined to be associated with subcutaneous administration of the CWHM-823 plus tGro- $\beta$ mixture (Supplemental Figure 1, A and B). Time and dose-response analysis revealed no increase in mobilization between $3 \mathrm{mg} / \mathrm{kg}$ and $15 \mathrm{mg} / \mathrm{kg}$ of CWHM-823, whereas peak mobilization was reached approximately 30 minutes after the injection (Supplemental Figure 1C).

Complementary to the testing of different VLA4 inhibitors, stimulation with tGro- $\beta$ (CXCL2) was compared with that of the alternative CXCR2 ligands CXCL1 (Gro- $\alpha$ ) and CXCL8 (IL-8). Again, all 3 agonists induced HSPC mobilization when given alone as well as in combination with CWHM-823 (Figure 1F). To control for specificity of the observed effects, CXCR2-KO mice were included. As expected, CXCR2 ligands alone did not induce mobilization in CXCR2-KO mice. Mobilization with the VLA4 antagonist was higher in absolute numbers yet qualitatively unchanged considering the higher baseline levels of circulating CFU-C (930 CFU-C/ $\mathrm{ml}[\mathrm{BALB} / \mathrm{cJ} \mathrm{CXCR} 2-\mathrm{KO}]$ versus $300 \mathrm{CFU} / \mathrm{ml}[\mathrm{BALB} / \mathrm{cJ} \mathrm{WT}]$ at baseline, and $3800 \mathrm{CFU}-\mathrm{C} / \mathrm{ml}$ [BALB/cJ CXCR2-KO]versus 1300 CFU-C/ml [BALB/cJ WT] mobilized with CWHM-823). Surprisingly, a decrease in mobilization with CWHM-823 was observed in CXCR2-KO mice when CXCR2 ligands were coadministered with the VLA4 antagonist. One possible explanation for this is that the bioavailability of CWHM-823 is reduced upon administration in conjunction with the chemokine as compared with its administration alone (Supplemental Figure 1B). Lack of specificity for the target receptor CXCR2 appears unlikely: in our comprehensive screening of $t$ Gro- $\beta$ against a panel of 348 different $G$ protein-coupled receptors, no cross-reactivity of the chemokine with any receptors other than CXCR2 was found (Supplemental Table 2).

Properties of mobilized cells. Having established that VLA4 inhibition combined with CXCR2 stimulation achieves superior CFU-C mobilization, we compared the repopulating capacity of the grafts mobilized with this new regimen, the single agents, or G-CSF (Figure 2A). For both VLA4 antagonists tested (firategrast and CWHM823), a significantly increased blood graft-derived contribution in the primary recipients was detected when each antagonist was combined with tGro- $\beta$ compared with engraftment from blood mobilized by either of the VLA4 antagonists or tGro- $\beta$ alone (Figure $2 \mathrm{~B}$ ). More importantly, despite higher numbers of CFU-Cs mobilized by G-CSF (Supplemental Figure 2 and data not shown), the concentration of repopulating units in the blood of mice mobilized with VLA4 antagonist plus tGro- $\beta$ was equal to that of G-CSF. Analysis of secondary recipients (i.e., evidence of serially repopulating units) confirmed virtually identical mobilization potency within the immature HSC fraction for G-CSF and VLA4 antagonist plus tGro- $\beta$ (Figure $2 \mathrm{C})$. Moreover, in a model of diabetes-associated poor mobilization $(10,35,36)$ generated by exposure of mice to the pancreas toxic agent streptozotocin, mobilization was better preserved with VLA4 antagonist plus tGro- $\beta$ combination as compared with G-CSF (7200 CFU-C/ml in diabetic compared with 22,200 CFU-C/ml in healthy mice with G-CSF; 9000 compared with $6350 \mathrm{CFU}-\mathrm{C} / \mathrm{ml}$ with CWHM-823 plus tGro- $\beta$, Supplemental Figure 2).

We then performed gene expression profiling of differentially mobilized HSPCs. LSK cells isolated from the blood of mice treated with AMD3100 (1 hour), G-CSF (5 days), or CWHM-823 plus tGro- $\beta$ (30 minutes) along with LSK cells from BM of untreated 
A

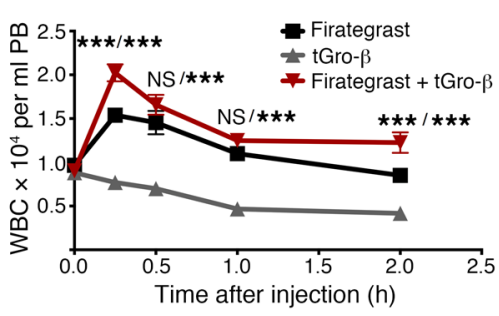

C

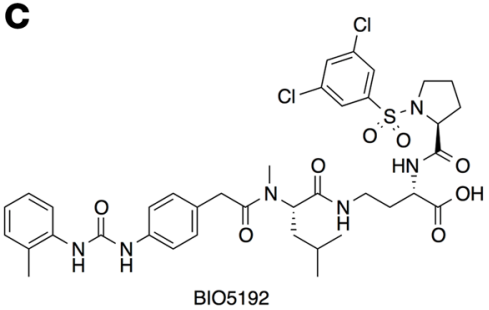<smiles>COc1cc(CC(=O)Nc2cc(CCC(O)O)on2)ccc1NC(=O)Nc1ccccc1C</smiles>

CWHM-842 (CP-664511; BIO5192 analog)
B

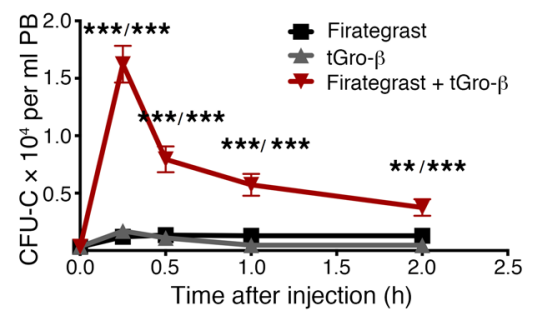<smiles>CCOCc1cc(OC)c(-c2ccc(CC(NC(=O)C=C(C)C)C(=O)O)cc2)c(OC)c1</smiles>

Firategrast

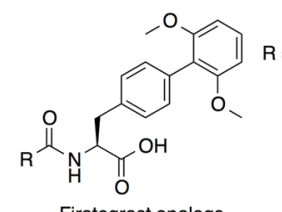

D

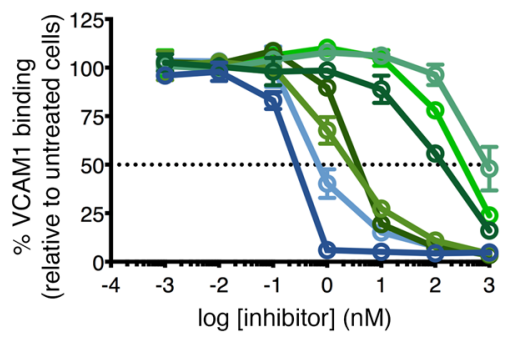

BlO5192 $\left(\mathrm{IC}_{50}=0.5 \mathrm{nM}\right)$

Firategrast $\left(\mathrm{IC}_{50}=230 \mathrm{nM}\right)$

- $\mathrm{CWHM}-842\left(\mathrm{IC}_{50}=0.9 \mathrm{nM}\right)$

○) CWHM-822 $\left(\mathrm{IC}_{50}=960 \mathrm{nM}\right)$

- $\mathrm{CWHM}-823\left(\mathrm{IC}_{50}=4.9 \mathrm{nM}\right)$

- $\mathrm{CWHM}-824\left(\mathrm{IC}_{50}=6.1 \mathrm{nM}\right)$

○. CWHM-825 $\left(\mathrm{IC}_{50}=570 \mathrm{nM}\right)$

E

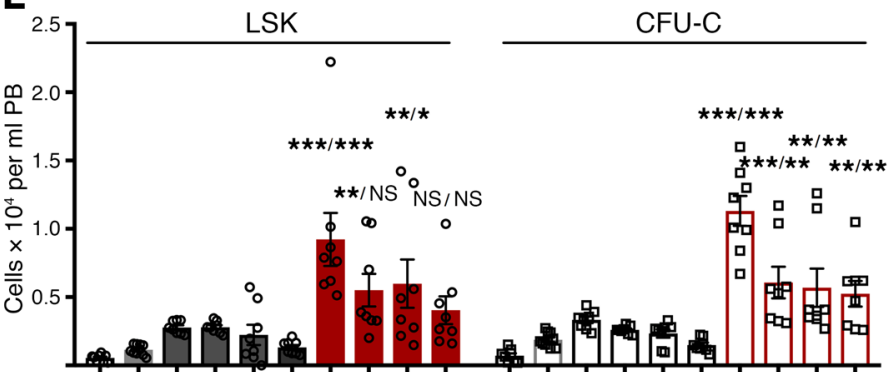

(x)
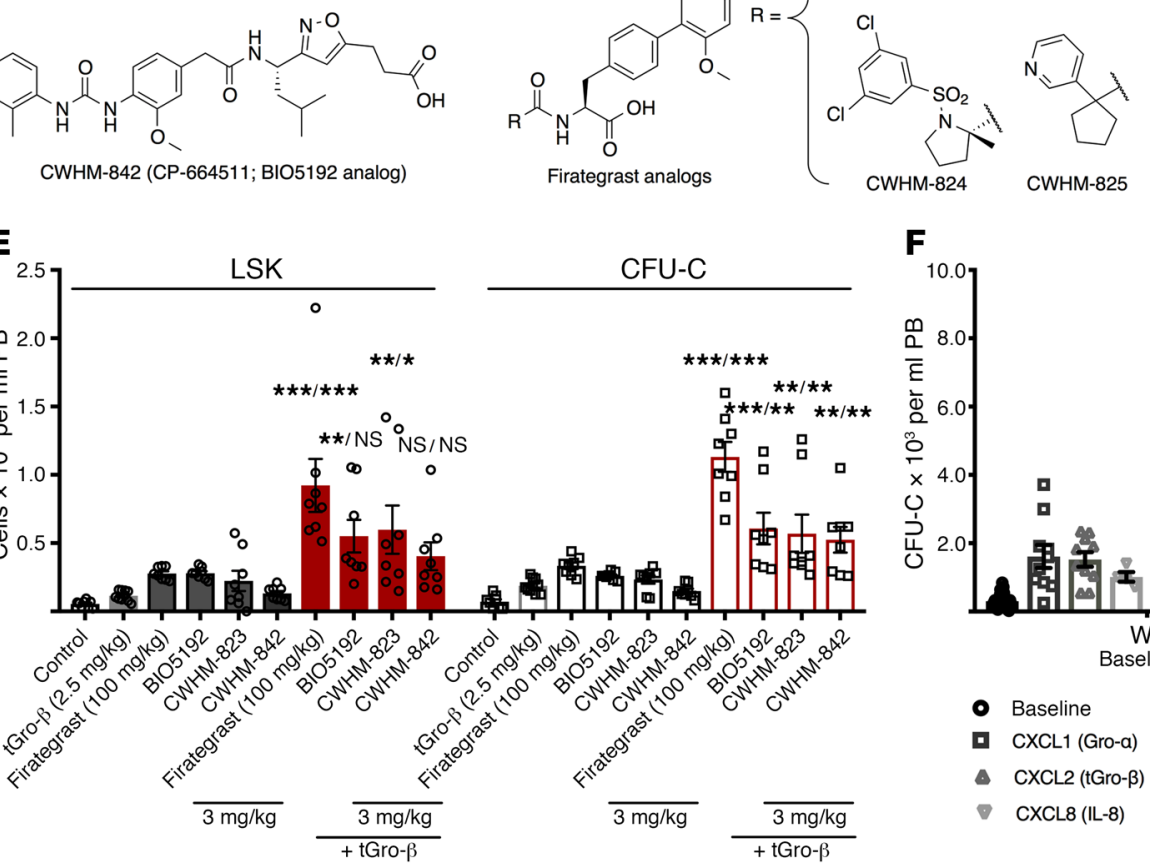

$\mathbf{F}$

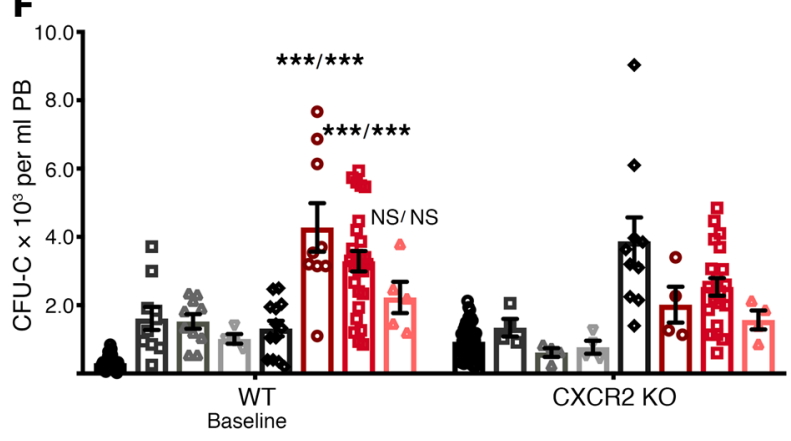

- Baseline CWHM-823

- $\operatorname{CXCL1}($ Gro-a) 0 CWHM823 + CXCL1 (Gro-a)

- CXCL2 (tGro- $\beta$ - CWHM823 + CXCL2 (tGro- $\beta$ )

- CXCL8(IL-8) CWHM823+ CXCL8 (IL-8)

Figure 1. Targeting VLA4 and CXCR2 to mobilize HSPCs. (A-B) DBA2/J mice were treated with the VLA4 inhibitor firategrast (100 mg/kg, i.v.), the CXCR2 ligand tCro- $\beta\left(2.5 \mathrm{mg} / \mathrm{kg}\right.$, s.c.) or both agents immediately after each other. Blood was analyzed for WBCs (A) and CFU-Cs (B). Data are mean \pm SEM, $n=5$. ${ }^{* * *} P<0.001$, ${ }^{* *} P<0.01$, compared with firategrast alone/compared with tCro- $\beta$ alone. (C) Molecular structures. (D) G2-ALL cells were treated in duplicate with the VLA4 inhibitors shown in C. Percent inhibition of VCAM1 binding as compared with untreated samples. Data are mean \pm SEM of a single experiment representative of 3 experiments. (E) DBA2/J mice were injected with tGro- $\beta$ (2.5 mg/kg, s.c.), a VLA4 antagonist (3 mg/kg, i.v., for BIO5192, CWHM-823, and -842; $100 \mathrm{mg} / \mathrm{kg}$, i.v., for firategrast), or their combination. Controls received vehicle only. Numbers of circulating CFU-Cs and LSK cells were analyzed 0.5 hours after the injection(s). Data are mean \pm SEM, $n=8-10 .{ }^{* *} P<0.001,{ }^{* *} P<0.01,{ }^{*} P<0.01$, compared with tGro- $\beta$ alone/VLA4 antagonist alone. (F) HSPC mobilization in CXCR2-KO mice using the CXCR2 ligands CXCL1, CXCL2 (tGro- $\beta$ ), and CXCL8 and the VLA4 antagonist CWHM-823 as well as their combinations was compared with that in WT BALB/C). Blood CFU-C numbers were analyzed at baseline, 15 minutes after injection of CXCR2 ligands (s.C., $1 \mathrm{mg} / \mathrm{kg}$ CXCL1 and CXCL8, $2 \mathrm{mg} / \mathrm{kg}$ tGro- $\beta$ ), 1 hour after injection of CWHM-823 (s.c., 3 $\mathrm{mg} / \mathrm{kg}$ ), and 30 minutes after the combined treatment (s.c. injection of each ligand together with CWHM-823 at same doses as single treatments). Data are mean \pm SEM, $n=4-26$ in mobilized groups, $n=51-78$ in baseline groups. ${ }^{* * *} P<0.001$, compared with CXCR2 agonist alone/compared with CWHM-823 alone. Statistical comparisons were made using linear mixed models in $\mathbf{A}$ and $\mathbf{B}$ and ANOVA in all others, followed by step-down Bonferroni's adjustment for multiple comparisons.

mice were examined using microarray analysis. A high degree of similarity between the analyzed LSK types was found, with AMD3100-mobilized LSK cells being the only group clustering somewhat separately from the other 3 using principal component analysis (PCA, Figure 2D). In comparison to BM, LSK cells mobilized by CWHM- 823 plus tGro- $\beta$ had only 45 genes with significantly different expression levels (Supplemental Table 3).
CWHM-823 plus tGro- $\beta$ versus G-CSF had only 53 such genes (Supplemental Table 4). By contrast, 694 genes were significantly up- or downregulated between AMD3100 and CWHM-823 plus tGro- $\beta$-mobilized LSK cells despite their very similar kinetics of mobilization (Supplemental Table 5). The close relationship between CWHM-823 plus tGro- $\beta$-mobilized LSK cells, BMresident LSK cells, and G-CSF LSK cells was further evident upon 
A

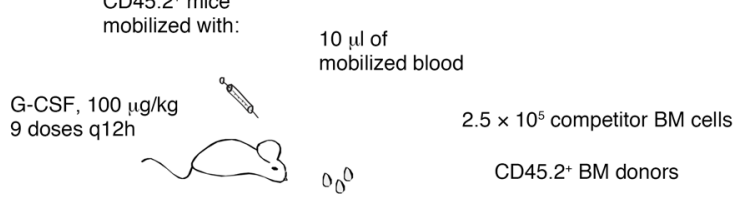

tGro- $\beta$

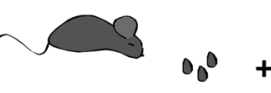

VLA4 antagonist

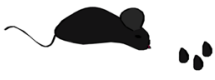

VLA4 antagonist

+ tGro- $\beta$

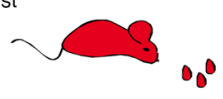

\section{C}

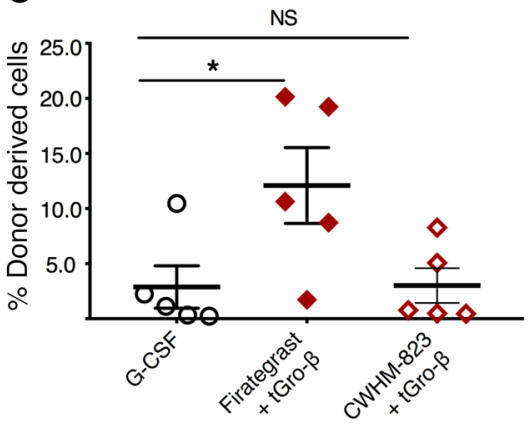

D

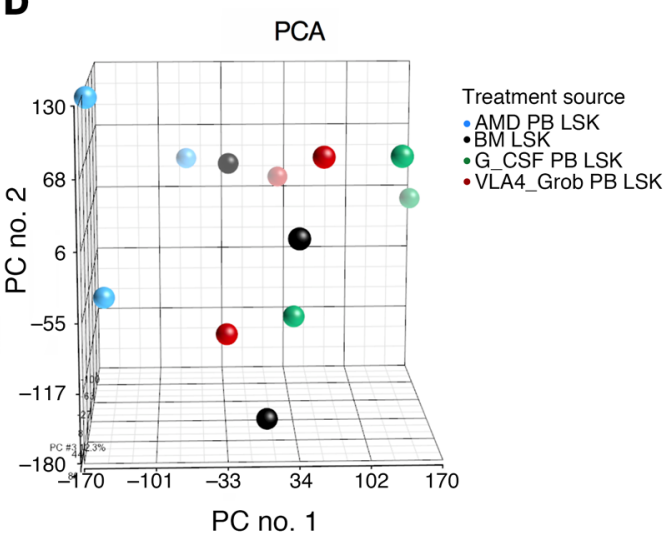

B

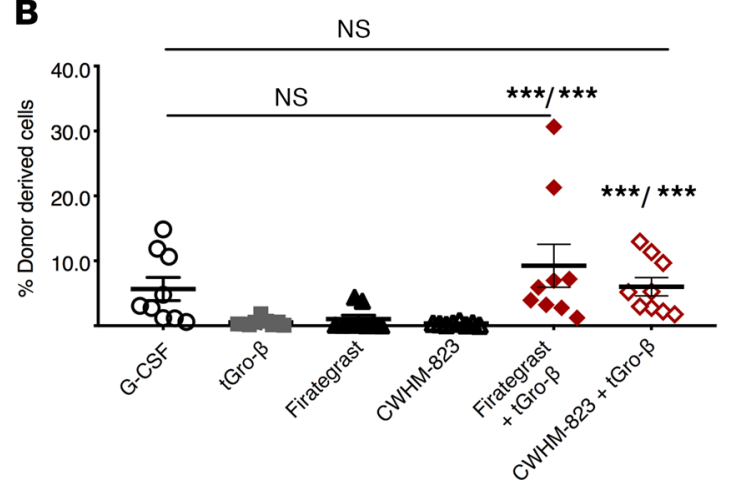

$\mathbf{E}$

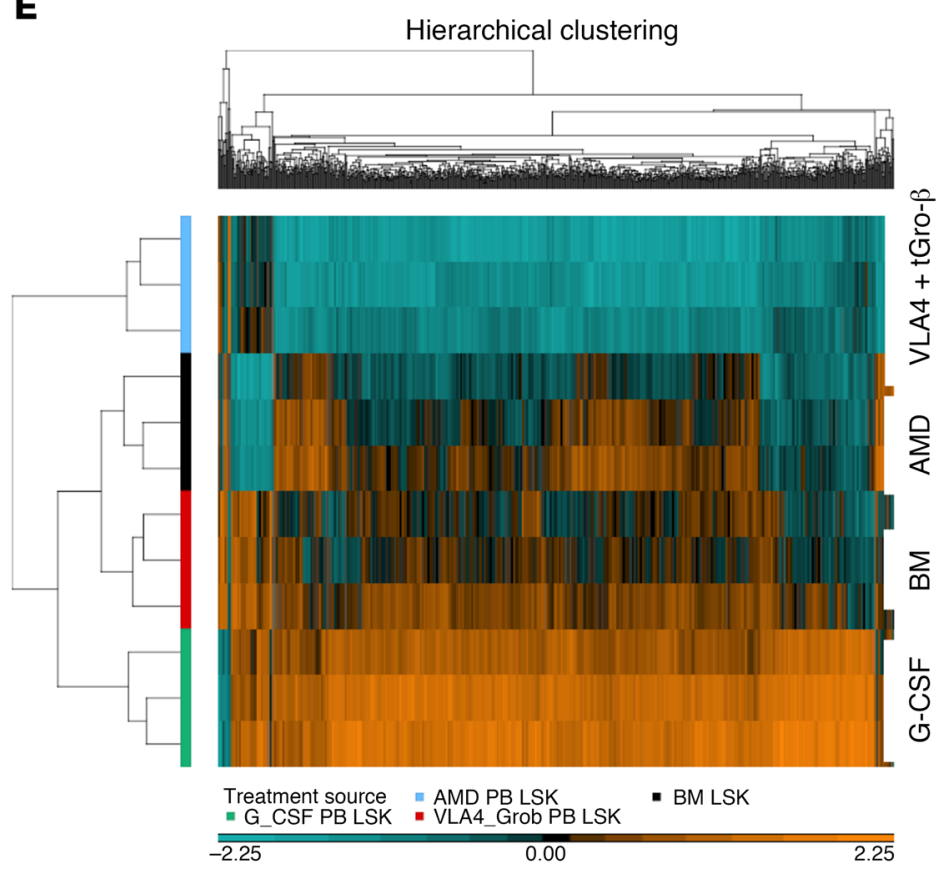

\section{F}

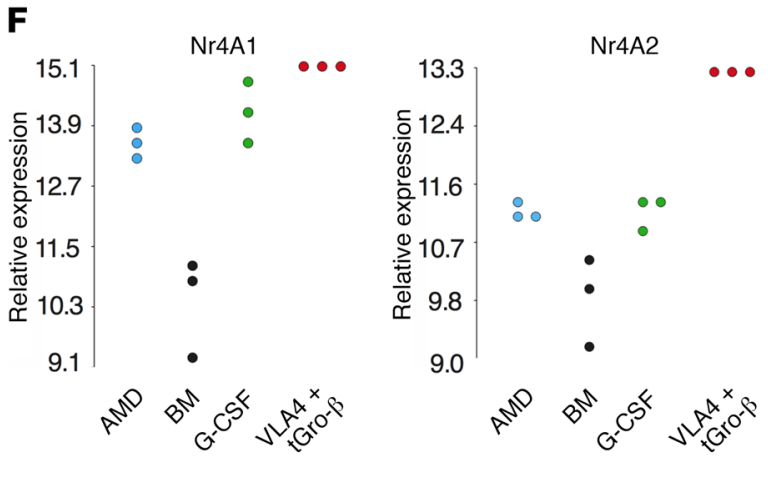

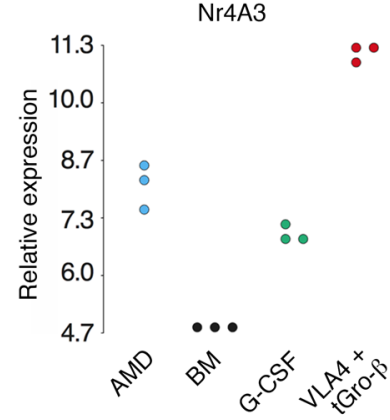


Figure 2. Properties of mobilized HSPCs. (A) Schema for analyzing competitive repopulating capacity. Blood $(10 \mu \mathrm{l})$ from $\mathrm{CD} 45.1^{+}$donors (BALB/c], $n=2-3$ ) mobilized with G-CSF (9 doses every 12 hours, $100 \mu \mathrm{g} / \mathrm{kg}$ per dose), tGro- $\beta$ (2.5 $\mathrm{mg} / \mathrm{kg}, 15$ minutes after s.c. injection), VLA4 antagonist firategrast (100 mg/ $\mathrm{kg}, 1$ hour after s.c. injection), and CWHM-823 (3 mg/kg, 1 hour after s.c. injection), or the combination of tGro- $\beta$ and VLA4 antagonist (dosed as indicated for single injections, 30 minutes after simultaneous injection) was mixed with CD45. $2^{+}$competitor BM cells (BALB/C], $n=2$ donors, $2.5 \times 10^{5}$ cells per recipient) and transplanted into lethally irradiated primary $\operatorname{CD} 45.2^{+}$hosts (BALB/C), $n=$ 8-10 recipients). (B) Percent CD45.1 $1^{+}$donor cells within the $C D 45^{+} C D 3^{-}$compartment of blood was evaluated 20 weeks after transplantation. Data are mean \pm SEM, $n=8-10 .{ }^{* *} P<0.001$ compared with VLA4 antagonist alone/compared with tCro- $\beta$ alone. (C) BM from the primary recipients was harvested, pooled, and transplanted into lethally irradiated secondary recipients (CD45.2+ BALB/ c), $2.5 \times 10^{6}$ per recipient). The percentage of donor-derived cells in the blood of secondary recipients 18 weeks after transplantation is shown. Data are mean \pm SEM, $n=5$ and are not significantly different. ${ }^{*} P<0.05$. (D-F) LSK cells were sorted from the blood of DBA/2) mice mobilized with G-CSF ( 9 doses every 12 hours, $100 \mu \mathrm{g} / \mathrm{kg}$ per dose, $n=3$ ), AMD3100 ( $5 \mathrm{mg} / \mathrm{kg}, 1$ hour after injection, $n=3$ ), or CWHM- 823 plus tGro- $\beta$ ( $3 \mathrm{mg} / \mathrm{kg}$ and $2.5 \mathrm{mg} / \mathrm{kg}$, 30 minutes after simultaneous s.c. injection, $n=3$ ). LSK cells from steady-state BM were included as control. Total RNA from sorted cells was subjected to microarray expression analysis. (D) Principal component analysis (PCA) of mRNA expression of different LSK species. (E) Corresponding hierarchical clustering map. (F) Normalized expression values for the genes Nr4A1-3. Each source/treatment group included $n=3$ samples (pooled from up to 6 donors). Statistical comparisons were made using ANOVA, followed by step-down Bonferroni's adjustment for multiple comparisons.

clustering of the top 1000 differentially expressed genes, which demonstrated clear separation of the AMD3100-mobilized LSK cells (Figure 2E).

Among the genes significantly enriched in CWHM-823 plus tGro- $\beta$-mobilized LSK cells, the transcription factors Nr4A1-3 were identified (Figure 2F). Members of this nuclear receptor family have previously been shown to specify a population of myeloid-biased long-term repopulating HSCs (37). This is consistent with a relative enrichment of the latter within CWHM-823 plus tGro- $\beta$-targeted HSPCs. Distinct properties of CWHM-823 plus tGro- $\beta$ LSK cells were further confirmed when cell cycle status of differentially mobilized HSPCs was assessed (Supplemental Figure 3). LSK cells mobilized with CWHM-823 were predominantly quiescent with more than $70 \%$ found in the $G_{0}$ phase and approximately $25 \%$ and $3 \%$ found in $G_{1}$ and $G_{2} / S / M$ phases of the cell cycle, respectively. A much lower proportion of tGro- $\beta-$ mobilized LSKs were not cycling (approximately $50 \%$ in $G_{0}$ ) and virtually no cells were in $\mathrm{G}_{2} / \mathrm{S} / \mathrm{M}$ phase. In contrast, more than half of the LSK cells from CWHM-823 plus tGro- $\beta$-mobilized blood were actively cycling and $5 \%$ was found in the $\mathrm{G}_{2} / \mathrm{S} / \mathrm{M}$ phase. Thus, LSK cells mobilized with the combination of VLA4 inhibition and CXCR2 stimulation do not simply represent a mix of those mobilized by single agents. Given the rapid kinetics of mobilization by these agents when administered alone and in combination, the discrepancies between the cell cycle distribution imply preferential targeting of distinct LSK species rather than changes induced upon exposure to the different agents.

Cellular mechanism of mobilization. We sought to determine which of the 2 events, CXCR2 stimulation or VLA4 inhibition, needs to occur first and is therefore the priming event for the subsequent response to the second stimulus. Pretreatment with the
VLA4 antagonist (CWHM-823, 45 minutes prior to tGro- $\beta$ administration) did not alter subsequent tGro- $\beta$-induced mobilization (Figure 3A). In contrast, albeit lower than when both compounds were given simultaneously, significantly elevated numbers of circulating CFU-Cs were detected in CWHM-823-mobilized mice that had been pretreated with $t$ Gro- $\beta$ as compared with PBS. These findings pointed toward CXCR2 targeting as the priming event.

Within the hematopoietic compartment, CXCR2 expression was detected almost exclusively in granulocytes, and specifically HSCs themselves were CXCR2-negative (Supplemental Figure 4). Therefore, as expected, when granulocytes were depleted from the circulation (Figure 3, B and C), mobilization responses to tGro- $\beta$ alone as well as to the combination of CWHM- 823 plus tGro- $\beta$ were completely blunted (Figure 3D).

In order to distinguish between hematopoietic-intrinsic versus nonhematopoietic contributions for mobilization with CWHM823 plus tGro- $\beta$, chimeric animals were generated by transplanting CXCR2-KO or WT BM cells into WT recipients and vice versa, WT cells into CXCR2-KO or WT recipients (Figure 4A). Following reconstitution, mice were mobilized with CWHM-823 alone, tGro- $\beta$ alone, or the combination. As expected, mobilization with the VLA4 antagonist alone was similar in both hematopoietic and stromal CXCR2 knockouts (Figure 4B). No HSPC mobilization with tGro- $\beta$ alone was observed in WT recipients reconstituted with CXCR2-KO BM (Figure 4C, first and second bars from left). Surprisingly, CXCR2-KO recipients engrafted with the WT BM also demonstrated attenuated mobilization (Figure 4C, fifth and sixth bars from left), indicating an additional contribution of nonhematopoietic (stromal) CXCR2 to the tGro- $\beta$-induced HSPC egress. Moreover, the combination of CWHM-823 and tGro- $\beta$ mobilized HSPCs with almost equal potency in WT recipients of CXCR2KO and WT BM (Figure 4D, comparing second and fourth bars from left). Mobilization of similar magnitude was also detected in CXCR2-KO and WT recipients reconstituted with WT BM. Stimulation of either hematopoietic or stromal CXCR2 was therefore sufficient to elicit synergistic mobilization when combined with inhibition of VLA4 signaling.

Expression and functional role of CXCR2 in endothelial cells (ECs) has been described $(38,39)$. Our previous studies indicated the absence of CXCR2 expression in CXCL12-abundant reticular cells (CARs, another major cellular player involved in HSPC retention) $(40,41)$. We therefore tested whether CXCR2 ablation restricted to ECs would lead to changes in mobilization responses. As shown in Figure 4E, mobilization with all 3 regimens (CWHM823 , tGro- $\beta$, and CWHM-823 plus tGro- $\beta$ ) was decreased in CXCR2 EC-KO recipients compared with the control group. Most pronounced was the effect observed with tGro- $\beta$ alone.

Role of cellular adhesion. Our studies suggested that stimulation of CXCR2 on either granulocytes or stroma was sufficient to recruit as many HSPCs into the circulation as when the target receptor was present in both compartments (Figure 4). This led us to investigate the role of adhesive cross-stimulatory interaction between neutrophils and stroma, likely initiated through exposure to the chemokine. Beta 2 integrins LFA1 (ITGAL/ITGB2, CD11a/ CD18) and Mac1 (ITGAM/ITGB2, CD11b/CD18) are well-known crucial mediators of neutrophil adhesion to vasculature $(42,43)$. Both have been shown to become activated after CXCR2 stimula- 
A

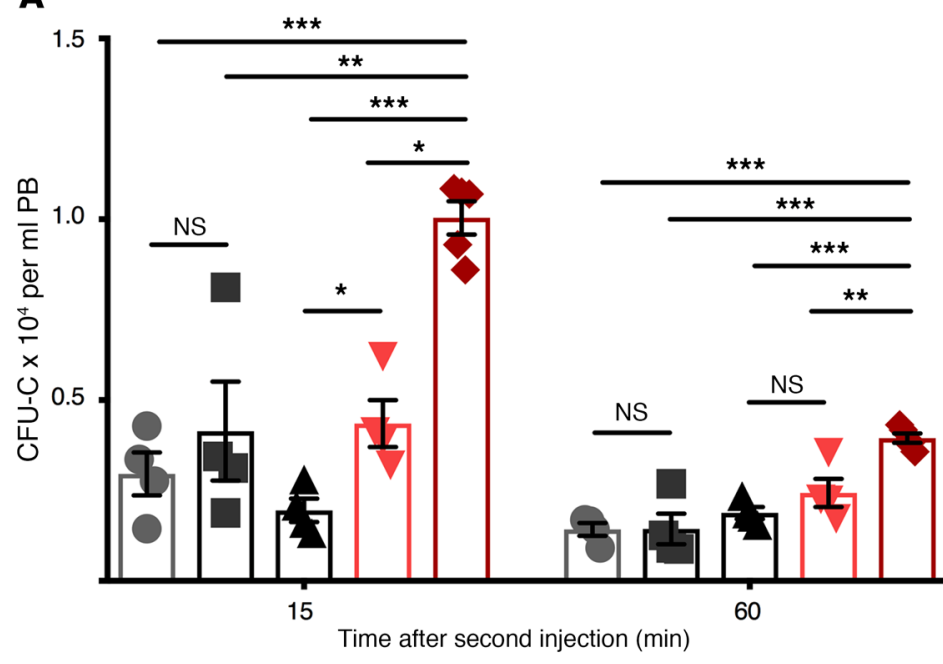

B
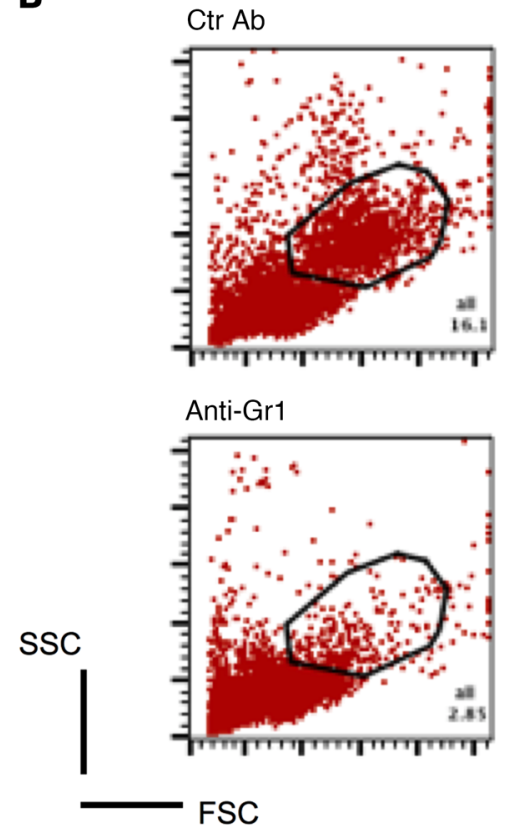

C

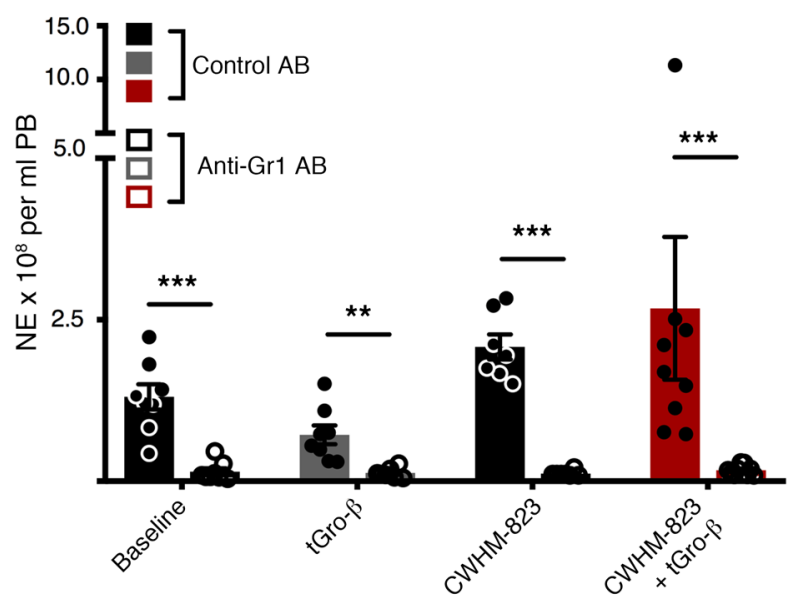

D

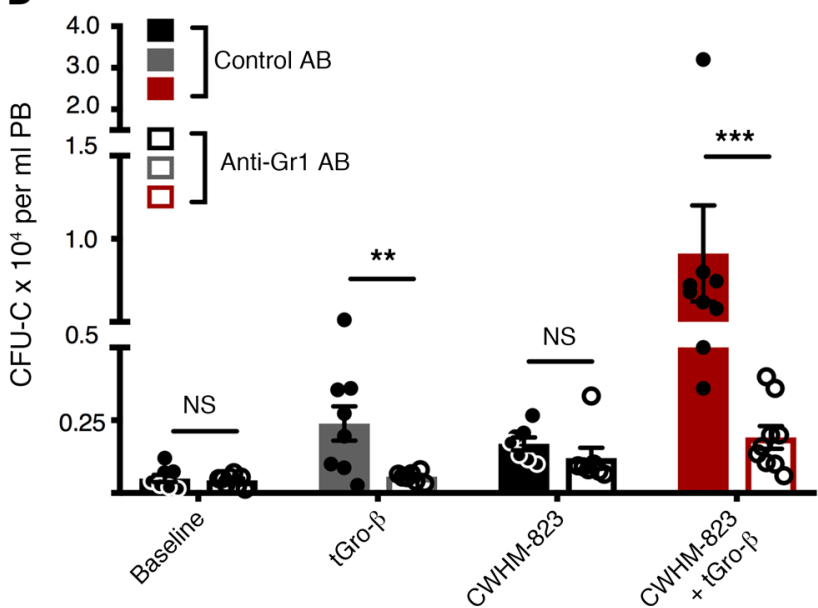

Figure 3. Granulocytes are indispensable for tGro-induced mobilization. (A) Effect of different sequences of injection. Two groups of BALB/c) mice received pretreatment with PBS or tGro- $\beta$ ( $2.5 \mathrm{mg} / \mathrm{kg}$, s.c.) 15 minutes prior to the injection of CWHM- 823 (3 mg/kg, s.c.). Accordingly, 2 other groups were pretreated with PBS or the VLA4 antagonist ( $3 \mathrm{mg} / \mathrm{kg}$ CWHM-823, s.c.) 45 minutes prior to tGro- $\beta$ ( $2.5 \mathrm{mg} / \mathrm{kg}$, s.c.) injection. A fifth group received a simultaneous injection of CWHM-823 plus tGro- $\beta$ at the indicated doses. At 15 and 60 minutes after the administration of the second compound (or the simultaneous injection in the control group), circulating CFU-C numbers were measured. Each bar is mean \pm SEM, $n=4-5 .{ }^{* *} P<0.001,{ }^{* *} P<0.01,{ }^{*} P<0.05$. (B-D) For transient depletion of blood neutrophils, mice were pretreated with an anti-Gr1 antibody in vivo (200 $\mu \mathrm{g} / \mathrm{mouse}$, i.v.) 36 hours prior to mobilization with tGro- $\beta$ ( 2.5 mg/kg, s.c., time point 15 minutes), CWHM-823 (3.0 mg/kg, s.c., time point 60 minutes), or the combined agents (doses same as for separate treatments, time point 30 minutes). Nonmobilized mice were used as controls (baseline). Moreover, all 4 conditions were analyzed in cohorts that had been pretreated with a control antibody (200 $\mu \mathrm{g} /$ mouse, i.v.). (B) Representative scatter plot analyses of unmobilized blood using flow cytometry, with granulocytes virtually absent in anti-Gr1-treated mice. (C-D) Numbers of circulating neutrophils (C) and CFU-Cs (D). Each bar is mean \pm SEM, $n=7-9$. ${ }^{* * *} P<0.001$, ${ }^{* *} P<0.01$. Statistical comparisons were made using ANOVA, followed by step-down Bonferroni's adjustment for multiple comparisons. Logarithm transformation was performed for the data in $\mathbf{C}$ and $\mathbf{D}$.

tion (44-46) and have been previously implicated in CXCR2- and G-CSF-triggered mobilization (19, 47, 48).

At baseline, no significant hematological changes were found in mice lacking LFA1 (CD11a-KO) or Mac1 (CD11b-KO) except for a slight increase in circulating neutrophils in CD11a knockouts (Figure 5, A-C). Mobilization of CFU-C with CWHM-823 was approximately doubled in CD11a- or CD11b-deficient mice, whereas both strains' response to tGro- $\beta$ alone was the same as in
WT mice, despite a dramatic neutrophilia induced in the CD11a$\mathrm{KO}$ mice in response to tGro- $\beta$ (Figure $5 \mathrm{~B}$ ). The response to the combination treatment was significantly attenuated (Figure $5 \mathrm{C}$ ).

Role of proteases. Considering the fact that CXCR2 is not expressed on the surface of the HSPCs themselves, we next addressed the question of the molecular crosstalk initiated by CXCR2 stimulation and how it enables HSPC egress. Previous reports aiming to elucidate the mechanism of tGro- $\beta$ and AMD3100 
A
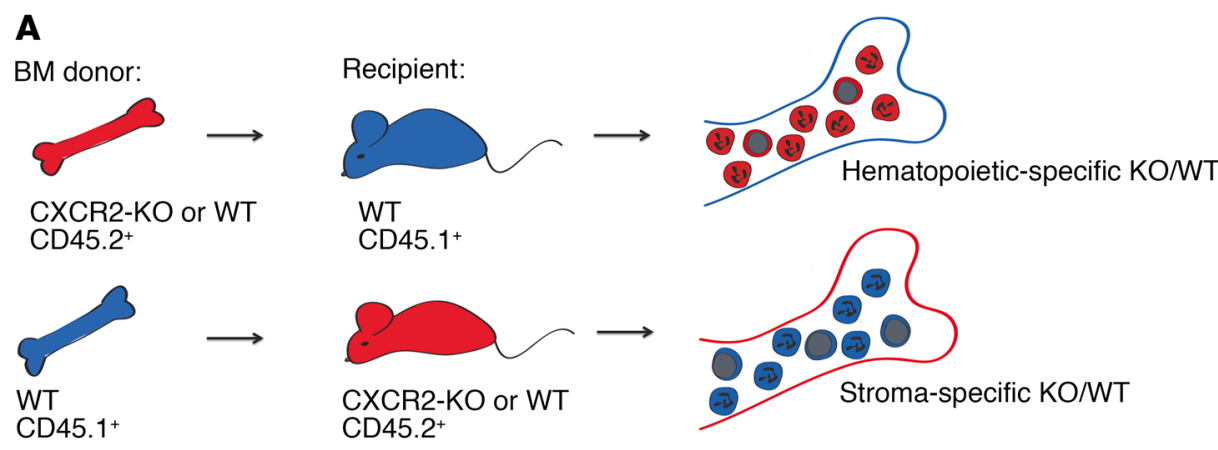

B

Baseline and CWHM-823 mobilization
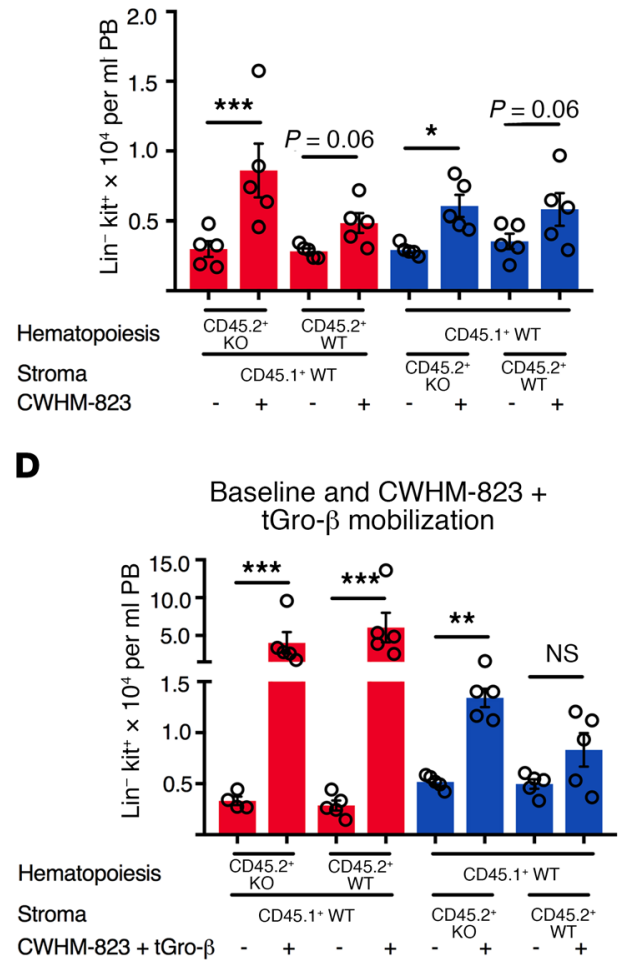

C

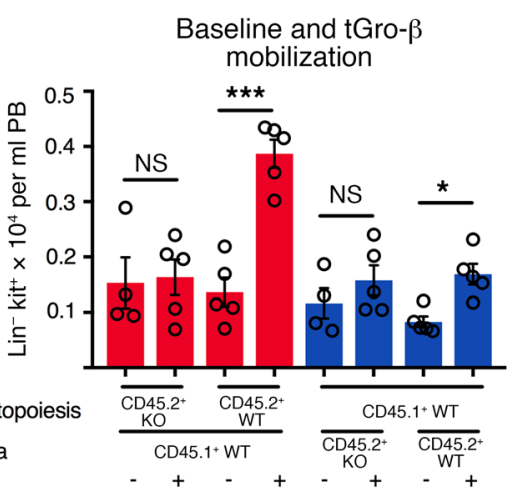

E

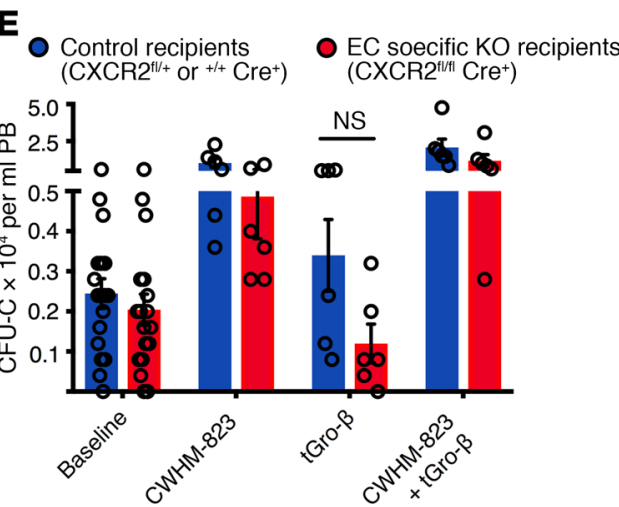

Figure 4. CXCR2 expression in both the hematopoietic and nonhematopoietic (stromal) compartment contributes to tGro-induced mobilization. (A) Hematopoietic-specific CXCR2-KO mice were generated by transplanting lethally irradiated CD45.1 $1^{+}$recipients (BALB/C)) with CD45.2 $2^{+}$BM cells isolated from CXCR2-KO mice (BALB/c, $1 \times 10^{6}$ cells per recipient, $n=3$ BM donor mice). A control group was reconstituted with $C D 45.2^{+}$WT BM cells (BALB/c, $1 \times 10^{6}$ cells per recipient, $n=3 \mathrm{BM}$ donor mice). Stromal-specific knockouts were generated by transplanting CD45.1 ${ }^{+}$WT BM cells (BALB/C), $1 \times 10^{6}$ cells per recipient, $n=3 \mathrm{BM}$ donors) into CXCR2-KO mice (CD45.2+). In the corresponding control group, WT CD45.2+ recipients were transplanted with the WT CD45.1+ BM graft. (B-D) Three months after transplantation, circulating HSPC numbers (Lin-kit ${ }^{+}$cells) were assessed in the different recipients at baseline and following mobilization with CWHM-823 alone ( $3 \mathrm{mg} / \mathrm{kg}, 1$ hour after s.c. injection, B), tGro- $\beta$ alone ( $2.5 \mathrm{mg} / \mathrm{kg}, 15$ minutes after s.c. injection, C), and both agents combined (dosed as indicated for separate treatments, 30 minutes after simultaneous s.c. injection, D). Each bar is mean $\pm \mathrm{SEM}, n=4-5$. ${ }^{* *} P<0.001,{ }^{*} P<0.01$, ${ }^{*} P<0.05$. (E) Lethally irradiated CXCR2 ${ }^{\mathrm{f} / \mathrm{fl}}$ Cdh5Cre+ hosts (C57BL/6 background, CD45.2+) were reconstituted with syngeneic WT CD45.1+ BM ( $3 \times 10^{6}$ cells per recipient) to generate EC-specific knockout recipients. In the control group, $\mathrm{CXCR2} 2^{\mathrm{fl} /+} \mathrm{Cre}^{+}$and $\mathrm{CXCR2}{ }^{+/+} \mathrm{Cre}^{+}$mice were used as recipients. Three months after transplantation, circulating HSPC (CFU-C) numbers were quantified at baseline and following mobilization with CWHM- 823 alone ( $3 \mathrm{mg} / \mathrm{kg}, 1$ hour after s.c. injection), tGro- $\beta$ alone ( $2.5 \mathrm{mg} / \mathrm{kg}, 15 \mathrm{minutes}$ after s.c. injection), and both agents combined (dosed as indicated for separate treatment, 30 minutes after simultaneous s.c. injection). Each bar is mean $\pm \mathrm{SEM}$, $n=6$. Statistical comparisons were made using ANOVA, followed by step-down Bonferroni's adjustment for multiple comparisons. Logarithm transformation was performed for the data in $\mathbf{D}$ and $\mathbf{E}$.

plus tGro- $\beta$-induced HSPC release suggested critical involvement of the matrix metalloprotease 9 (MMP9) (49-52). Therefore, we compared mobilization with the combination of CWHM-823 plus tGro- $\beta$ against AMD3100 plus tGro- $\beta$ as well as single agent treatments in MMP9-KO mice on both FVB and C57BL/6 (B6) background (Supplemental Figure 5, A and B). In addition, a pharma- cologic model in the form of a broad-spectrum MMP inhibitor, batimastat, was employed (Supplemental Figure 5C). Baseline circulating HSPC numbers or AMD3100-induced mobilization were not affected by the genetic MMP9 deficiency or by the MMP blockade. CWHM-823-induced mobilization was equally potent in FVB WT and FVB MMP9-KO mice and was not affected by bati- 
A

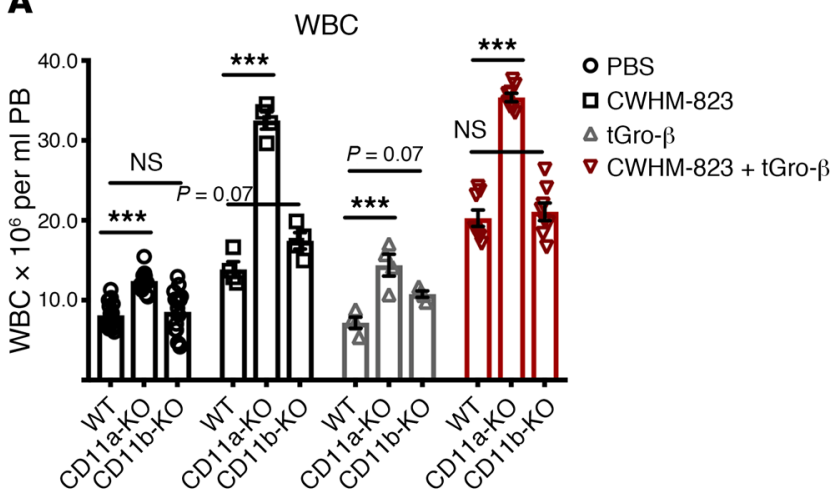

B

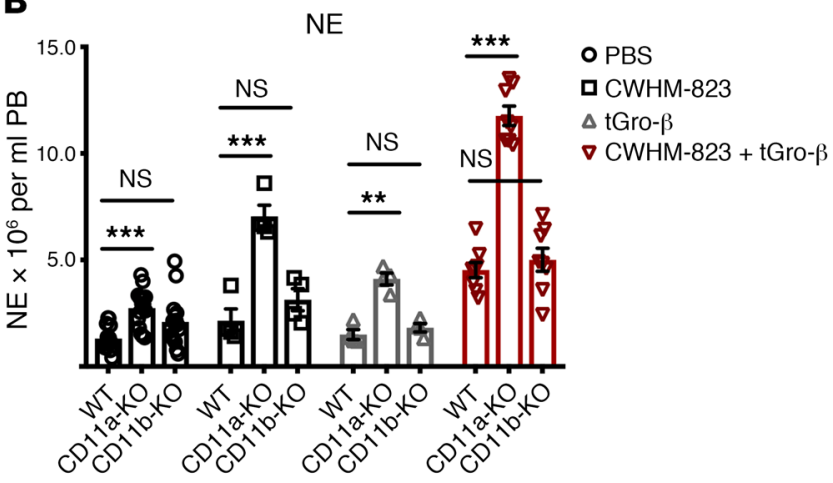

C

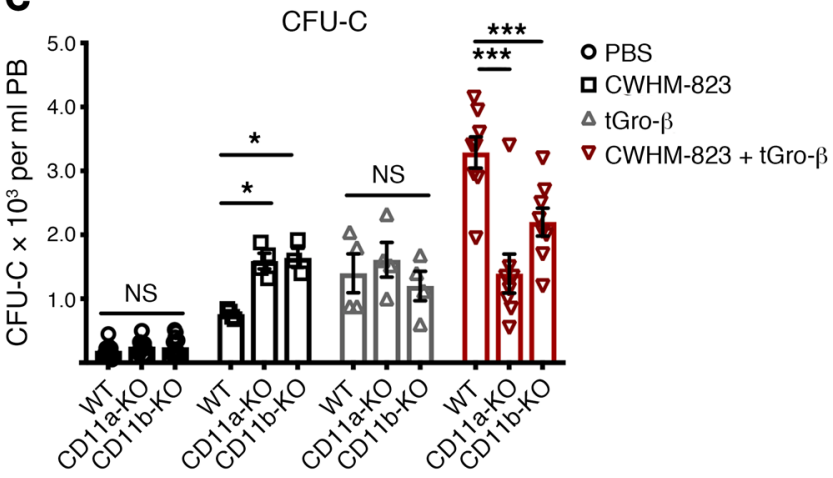

Figure 5. Role of cell adhesion on mobilization. (A-C) Mobilization in LFA1 (CD11a) and Mac1 (CD11b) knockout mice. CD11a-KO and CD11b-KO as well as WT C57BL/6) mice received an injection of PBS, tGro- $\beta$ ( $2.5 \mathrm{mg} / \mathrm{kg}$, s.c., time point 15 minutes), CWHM-823 (3.0 mg/kg, s.c., time point 60 minutes), or the 2 agents combined (dosed the same as for separate treatments, time point 30 minutes). Mobilization of WBCs (A), neutrophils (NEs) (B), and CFU-Cs (C) was assessed. Each bar is mean $\pm \mathrm{SEM}, n=4-8$. ${ }^{* * *} P<0.001,{ }^{* *} P<0.01,{ }^{*} P<$ 0.05 . Statistical comparisons were made using ANOVA, followed by step-down Bonferroni's adjustment for multiple comparisons.

mastat. However, mobilization was stronger in B6 MMP9-KO mice than in their WT counterparts. Conversely, tGro- $\beta$ - and AMD3100 plus tGro- $\beta$-induced mobilization was significantly reduced in FVB MMP9-KO mice or following MMP inhibitor treatment, but it was not altered in B6 MMP9-KO mice. Interestingly, the combination of CWHM-823 plus tGro- $\beta$ mobilized with equal efficiency in both knockout strains relative to WT control mice, whereas mobilization was slightly, but not significantly, reduced in mice pretreated with batimastat. Taken together, these data do not support a clear role of MMP9 in CWHM-823 plus tGro- $\beta$-associated augmented mobilization. Rather, they suggest differences in the mechanism underlying mobilization induced by combining CXCR2 stimulation and VLA4 blockade compared with CXCR2 activation in conjunction with CXCR4 blockade.

\section{Discussion}

In the current study, a novel mobilization strategy, CXCR2 stimulation combined with inhibition of the VLA4 integrin, was investigated and found to result in a rapid, synergistic, and highly efficient recruitment of HSPCs into the circulation. Implementation of VLA4 antagonists with improved properties allowed for comprehensive testing and optimization of mobilization achieved through VLA4 targeting. This revealed VLA4 as a promising target associated with mobilization of a clinically relevant magnitude. Compared with mobilization with G-CSF, long-term serially repopulating HSCs were relatively more strongly enriched in VLA4 inhibitor plus CXCR2 agonist-mobilized grafts than clonogenic cells without long-term repopulating capacity, indicating preferential targeting of a very immature HSPC fraction. Given how brisk the mobilization occurred and assuming BM sinusoids as the exit route for HSCs, our findings support the notion of a close proximity of the mobilization-sensitive HSCs to BM sinusoids. Moreover, the numbers of rapidly mobilizable HSCs markedly exceeded previous estimates. Furthermore, we found an unexpected contribution of stromal (endothelial) CXCR2 to the mobilization induced by tGro- $\beta$ alone as well as by VLA4 antagonist plus tGro- $\beta$, hinting at a previously unsuspected crosstalk between granulocytes and stroma as a master regulator of HSPC localization and egress.

The cytokine G-CSF and chemotherapy-triggered myeloid rebound, the 2 commonly utilized clinical mobilization regimens, are comprised of 2 partly overlapping steps, proliferation and the actual release of the HSPCs from the BM $(53,54)$. Although not necessary nor sufficient for mobilization per se, proliferation (and therefore expansion) of HSPCs clearly contributes to the magnitude of G-CSF and chemotherapy-induced HSPC mobilization (55). In addition to the obvious increase in numbers, HSPC localization has been reported to change over the course of proliferation, favoring closer proximity to the vessels, which in turn facilitates $\mathrm{BM}$ exit $(56,57)$. In comparison, the rapid kinetics of mobilization with VLA4 antagonist plus tGro- $\beta$ (peak reached within 15-30 minutes of s.c. injection) would not allow for cell division to occur prior to the HSPC release from the BM. Therefore, the relative enrichment for primitive, serially repopulating units in the VLA4 antagonist plus tGro- $\beta$-mobilized blood grafts likely reflects their preferential targeting and a preferential vascular (sinusoidal) localization. Compared with G-CSF and chemotherapy, much less severe alterations in the BM composition in the course of VLA4 antagonist plus tGro- $\beta$-mediated mobilization are to be expected, which is particularly important in light of the very similar potency.

Mature hematopoietic cells contained in the transplant have been shown to directly influence HSPC engraftment. For example, the presence of regulatory $\mathrm{T}$ cells was demonstrated to ensure HSPC survival and colonization of the host BM (58). Also, bystander effects of the myeloid compartment, granulocytes in particular, for the establishment of the niche following irradiation/transplantation were recently described (59). Thus, granulocyte-derived 
A

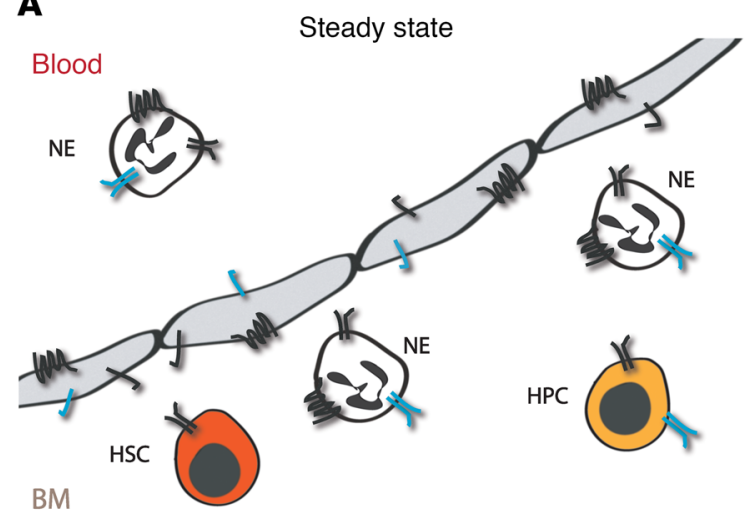

C

CXCR2 agonist alone

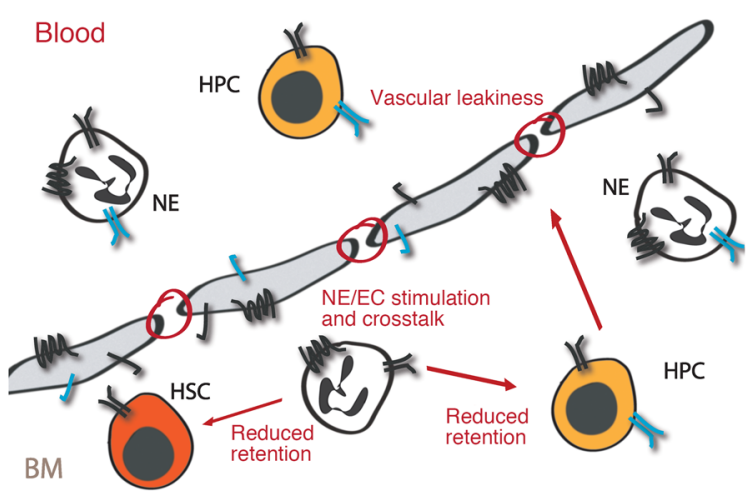

B

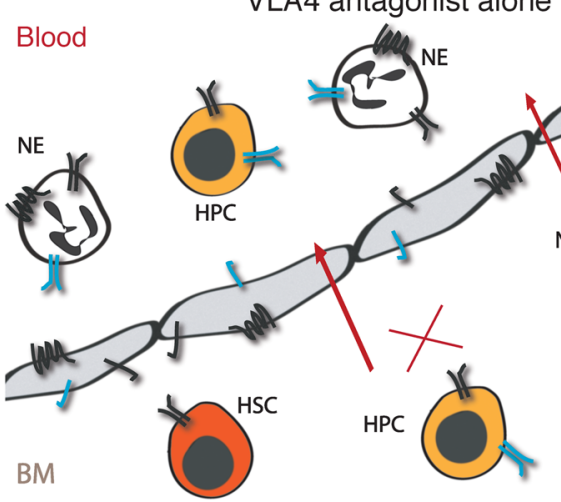

D

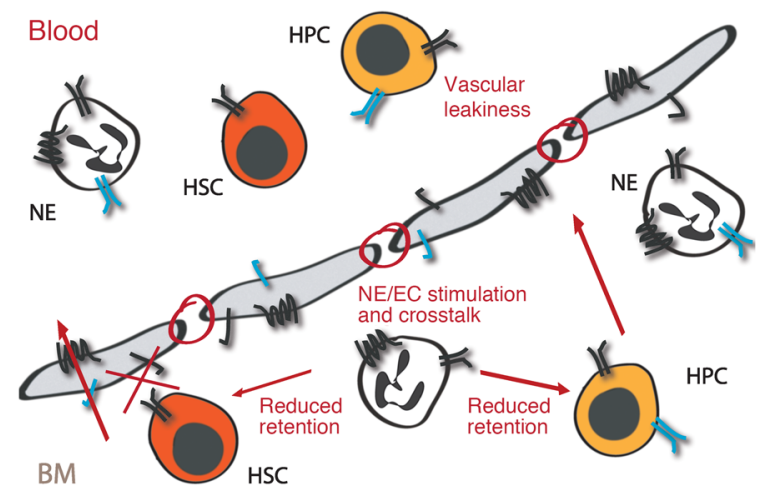

Y VLA4

M cXCR2

Beta2 integrin

ICAM1

VCAM1

Figure 6. Proposed mechanisms for targeting CXCR2 and VLA4 to mobilize hematopoietic stem cells. (A-D) Schematic presentation of HSPC and mature hematopoietic cell (NE) localization in the BM relative to the vasculature. Adhesive interactions and cellular distribution are shown under steady state (A) compared with following mobilization with a VLA4 antagonist (B), CXCR2 agonist (C), and the combination of the 2 strategies (D). For a detailed description see the Discussion.

TNF- $\alpha$ was shown to facilitate vasculogenesis, a critical step during regeneration. Given the high percentage as well as the direct stimulation of neutrophils in VLA4 antagonist plus tGro- $\beta$-mobilized (and also G-CSF-mobilized) blood specimens, it is conceivable that granulocyte-derived factors contribute to the superior performance of the corresponding grafts. While not excluding this possibility, the results of our LSK gene expression profiling further imply inherent differences in the profile and composition of the differentially mobilized versus BM resident HSPC populations themselves. In line with the idea that differences within the HSPC fraction (i.e., a higher proportion of more immature, serially repopulating cells) are primarily responsible for the observed graft fitness, LSK SLAM cells purified from AMD3100 plus tGro- $\beta$-mobilized blood (a regimen very similar to ours) were found to outperform those isolated from G-CSF-treated blood donors when both were tested head-to-head in a competitive transplant assay against BM cells (52).

Neutrophils have been demonstrated to be indispensable for IL-8-induced (and G-CSF-induced) mobilization (51, 60). Therefore, our observation of a significant reduction of tGro- $\beta$ as well as CWHM-823 plus tGro- $\beta$-associated mobilization in the context of granulocyte deficiency was in line with expectations. By contrast, the subsequent findings that nonhematopoietic CXCR2expressing cells coregulate HSPC egress triggered by tGro- $\beta$ alone and that the presence of the receptor in either one of the com- partments, stroma or hematopoietic cells, was in fact sufficient to elicit HSPC egress when the combination of CWHM-823 plus tGro- $\beta$ was used, were very much surprising. Thus, while the presence of granulocytes was necessary for both, tGro- $\beta$ and CWHM823 plus tGro- $\beta$-induced mobilization, lack of CXCR2 expression on their surface seemed, to a large extent, to be compensated for in the case of CWHM-823 plus tGro- $\beta$ treatment, as long as the stromal CXCR2 was present.

Relevant for the interactions between HSPC and neutrophils, a role of CXCR2 expression in endothelia has been increasingly recognized. Accordingly, CXCR2 expression in ECs of the lung vessels and its involvement in controlling neutrophil passage from the circulation into the lung parenchyma have been described (38). CXCR2 signaling has also been shown to critically mediate EC proliferation and therefore vascular repair after transplantation (39). Moreover, involvement of the zebrafish CXCR2 ortholog CXCR1 in the hematopoietic stem cell niche remodeling during embryogenesis has been demonstrated (61). Our findings along with the results of a companion study (unpublished observations) imply an additional, immediate role of endothelial CXCR2 in the regulation of HSPC retention versus egress.

Significant controversy surrounds the role of matrix metalloproteases in general and MMP9 in particular for mobilization regimens involving neutrophil stimulation such as tGro- $\beta$, IL-8, or 
G-CSF. In a recent study by Hoggatt et al. describing a mobilization approach similar to ours (AMD3100 plus tGro- $\beta$, ref. 52) the authors reported a contribution of MMP9 activity to the synergistic mobilization response in mice. Moreover, a correlation between mobilization efficiency in healthy G-CSF donors and activity of the MMP9 inhibitor TIMP1 was described. In our analysis, a trend toward a more pronounced contribution of MMP9 during HSPC egress induced by AMD3100 plus tGro- $\beta$ as compared with CWHM-823 plus tGro- $\beta$ was observed. However, a requirement for intact MMP9 for a tGro- $\beta$-based regimen could not be corroborated.

The differences in the magnitude and kinetics of HSPC egress associated with the different regimens analyzed in this study, as well as the distinct properties of cells mobilized with VLA4 antagonists alone, versus tGro- $\beta$ alone, versus VLA4 antagonist plus tGro- $\beta$, can be interpreted as a result of targeting distinct HSPC subsets (Figure 6A-D). Thus, based on their performance in the competitive transplantation setting (Figure $2, \mathrm{~A}-\mathrm{C}$ ), more mature progenitor cells are primarily mobilized with a VLA4 antagonist alone (Figure 6B). Expression of the beta 2 integrins LFA1 and Mac1 on human and murine hematopoietic progenitor cells (HPCs) but not stem cells has been demonstrated $(48,62)$. Hence, although not markedly dislodging stem cells when blocked individually, inhibition of LFA1 or Mac1 in conjunction with VLA4 blockade resulted in enhanced HPC mobilization. Consistent with the direct mode of action of VLA4 antagonists $(4,63)$, loss of granulocytes did not affect VLA4 inhibitor-induced HSPC egress.

Peak mobilization after tGro- $\beta$ treatment is remarkably fast. Activation of CXCR2 signaling in ECs has been reported to lead to EC contraction $(64,65)$ and weakening of intercellular junctions $(66,67)$. In accordance with these reports, we detected increased permeability of vascular cells after CXCR2 stimulation in vivo and in vitro (unpublished observations). Increased vascular leakiness has also been found in the BM of tGro- $\beta$-treated mice (52) and is likely a major contributor to the brisk kinetics of tGro- $\beta$-triggered HSPC emigration (Figure 6C). Moreover, though not addressed in our analysis directly, an involvement of the complement cascade, a key orchestrator of pharmacologically and pathologically induced HSPC release from the $\operatorname{BM}(68,69)$, and the associated release of anaphylatoxins might have facilitated increased vascular permeability. The latter are further known to stimulate the release of the lipolytic enzyme phospholipase $C \beta 2$ (PLCB2), which in turn disrupts the lipid raft formation needed for optimal signaling via VLA4 and CXCR4 $(68,70)$.

Adhesive interactions and cross-stimulation between CXCR2expressing granulocytes and endothelia are critical for the increased permeability and likely also for the subsequent cell-cell contact or soluble mediator-enabled HSPC release in the context of mobilization with tGro- $\beta$ alone. However, in contrast to the reported requirement of LFA1 for IL-8-induced mobilization (19), neither deficiency for LFA1 nor for Mac1 enhanced tGro- $\beta$ mediated HSPC egress. Loss of one beta 2 integrin was possibly compensated for by the presence of the other, along with other integrins. With regard to the HSPC population targeted, the transplant data suggested predominant mobilization of progenitor cells with tGro- $\beta$, as was the case with VLA4 antagonist alone. Hence, elevated vascular permeability and reduced retention induced by tGro- $\beta$ alone are not sufficient to release the HSCs from their protective BM environment.
Finally, when tGro- $\beta$ is combined with a second stimulus in the form of VLA4 inhibition, in addition to the reciprocal stimulation of CXCR2-expressing cells, the associated changes in permeability and cell contact and/or soluble mediator decreased HSPC retention, which is further hit by lack of VLA4 signaling (Figure 6D). As demonstrated by the results of the serial competitive transplantation analysis, this combination of events indeed recruited with high efficiency highly engraftable, true HSCs into the circulation. Cross-stimulation of tGro- $\beta$ targeted cells that were dependent on integrin interactions, and blockade of both VLA4 and either LFA1 or Mac1 appears to disturb rolling and adhesion and therefore proximity of CXCR2-expressing cells to an extent that cannot be compensated, unlike when tGro- $\beta$ is given alone. This concept could explain the relatively less efficient mobilization with VLA4 antagonist plus tGro- $\beta$ in mice lacking beta 2 integrins.

In summary, we show that simultaneous administration of CXCR2 agonists in conjunction with VLA4 small-molecule inhibitors results in rapid and robust mobilization of HPCs and HSCs. A contribution of hematopoietic (neutrophils) and nonhematopoietic (endothelial) CXCR2 to the mobilization response was found. Grafts mobilized with the VLA4 inhibitor plus tGro- $\beta$ combination exhibited serial repopulating capacity. Given several advantages of this combination over currently approved HSPC mobilization methods, it represents an exciting potential strategy for clinical development in the future. We are continuing to optimize the pharmacokinetic and pharmacodynamic properties of the VLA4 inhibitors to enable future large-animal efficacy and toxicology studies in nonhuman primates, which are currently underway. Further, our data support the proposed perisinusoidal localization of very primitive (true) HSCs and will also serve as a tool to learn more about the interplay between mature hematopoietic cells, nonhematopoietic stroma, and stem cells.

\section{Methods}

\section{Mice}

Male 6- to 12-week-old WT BALB/cJ (CD45.2+), BALB/cByJ (CD45.2+), and syngeneic CByJ.SJL(B6)-Ptprca/J (CD45.1+) as well as WT C57BL/6J and syngeneic B6.SJL-Ptprc ${ }^{a}$ Pep3 ${ }^{b}$ /BoyJ (CD45.1 ${ }^{+}$), WT $\mathrm{DBA} / 2 \mathrm{~J}$, and FVB/NJ mice were purchased from the Jackson Laboratory. The knockout strains C.129S2(B6)-Cxcr2tm1Mwm/J (CXCR2KO, BALB/cJ background), B6.FVB(Cg)-Mmp9tm1Tvu/J (MMP9-KO, C57BL/6J background), FVB.Cg-Mmp9tm1Tvu/J (MMP9-KO, FVB/ NJ background), B6.129S7-Itgaltm1Bll/J (CD11a-KO, C57BL/6J background), and B6.129S4-Itgamtm1Myd/J (CD11b-KO, C57BL/6J background) were also obtained from Jackson Laboratory. Endothelialspecific CXCR2 ablated mice were generated by crossing C57BL/6Cxcr2tm1Rmra/J (CXCR2 $2^{\mathrm{f} / \mathrm{fl}}$, Jackson Laboratory) and B6;129-Tg (Cdh5-cre)1Spe/J (Cdh5Cre, Jackson Laboratory) mice. Mobilization experiments were performed with $C x c r 2^{\mathrm{t} / \mathrm{l} / \mathrm{Cre}}{ }^{+}$mice $\left(\mathrm{CXCR} 2^{\mathrm{f} / \mathrm{WT}} \mathrm{Cre}^{+}\right.$ were used as controls) reconstituted with WT BM from syngeneic B6.SJL-Ptprc ${ }^{a} P e p 3^{b}$ /BoyJ (CD45.1 $1^{+}$, Jackson Laboratory) donors. Following lethal irradiation (1×9.5-11 Gy, using a cesium source) and transplantation, mice were kept on $0.5 \mathrm{mg} / \mathrm{ml}$ sulfamethoxazole and $0.1 \mathrm{mg} / \mathrm{ml}$ trimethoprim (HI-Tech Pharmacal), administered by mouth in drinking water. 


\section{Cell and tissue preparation}

Peripheral blood (PB) and BM cells were recovered as previously described (12). Cell counts (WBC) were measured on an automatic hemocytometer (Hemavet 950, Drew Scientific). Total counts (per tissue) as well as the majority of functional analysis were performed with filtered and otherwise unmanipulated cell suspensions, whereas red cells were removed from suspensions prepared for flow cytometry using hypotonic lysis. Absolute numbers of immunophenotypically defined populations (e.g., LSK cells) were calculated assuming $\mathrm{CD}_{4} 5^{+}$cells are equivalent to $\mathrm{WBC}$ from the corresponding cell count analysis.

\section{In vivo reagents and treatments}

Human recombinant truncated Gro- $\beta$ peptide (tGro- $\beta$, CXCL2, SB-251353 from GlaxoSmithKline) was used for all in vivo experiments. Stock solution stored at $-80^{\circ} \mathrm{C}$ was freshly thawed and diluted immediately prior to the s.c. injection $(2.5 \mathrm{mg} / \mathrm{kg})$ (52). Murine recombinant CXCL1 (PeproTech) and human recombinant CXCL8 (aa 23-99, Sino Biological) stock solutions were prepared in water. All chemokines were diluted in PBS (GE Healthcare Life Sciences) or used directly in the CWHM-823 solution. CWHM-823 plus chemokine mixtures were sonicated in a water bath for 15-30 minutes prior to injection to counteract precipitation. Firategrast was synthesized based on the published structure $\left(\mathrm{C}_{27} \mathrm{H}_{27} \mathrm{~F}_{2} \mathrm{NO}_{6}\right.$; patent no. US2014051655), dissolved in a $1 \%$ ethanol solution in PBS and injected i.v. or s.c. $(100 \mathrm{mg} / \mathrm{kg})$. Mice treated with firategrast plus tGro- $\beta$ received 2 separate injections. All other VLA4 antagonists, BIO5192 (TOCRIS, Bio-Techne Corporation) (28), CWHM-842, $-822,-823,-824$, and -825 were dissolved in DMSO (100× stock solution) and diluted in $\mathrm{NaHCO}_{3} / \mathrm{NaCl}$ buffer $\left(1: 1,10 \mathrm{mM} \mathrm{NaHCO}_{3}\right.$, $\mathrm{pH} 8 ; 0.9 \% \mathrm{wt} / \mathrm{vol} \mathrm{NaCl}$ ) for subsequent i.v., s.c. (CWHM-823 only), or i.p. (CWHM-823 only) injection at $3 \mathrm{mg} / \mathrm{kg}$. BIO5192 plus tGro- $\beta$ and CWHM-842 plus tGro- $\beta$ treatment consisted of 2 separate injections, whereas CWHM-823 plus Gro- $\beta$ was administered either as 2 injections (VLA4 antagonist i.v., tGro- $\beta$ s.c.) or as one injection (i.v., i.p., or s.c.) as specified for each experiment. AMD3100 (Mozobil, Genzyme) suspension was prepared in PBS and administered s.c. $(5 \mathrm{mg} / \mathrm{kg})$. AMD3100 plus tGro- $\beta$ mixture was prepared by diluting tGro- $\beta$ in the AMD3100 solution and then s.c. injected. RhG-CSF (Neupogen, Filgrastim, Amgen) diluted in PBS was injected i.p. every 12 hours at a dose of $100 \mu \mathrm{g} / \mathrm{kg}$ for a total of 4 (day 3) or 9 (day 5) doses. Broad spectrum matrix metalloprotease inhibitor batimastat (BB-94, APExBio) was dissolved in DMSO, diluted in corn oil (Sigma-Aldrich), vortexed thoroughly, and injected i.p. 12 and 2 hours $(25 \mathrm{mg} / \mathrm{kg})$ prior to the mobilization treatment. Control mice received DMSO/corn oil injections. In all other experiments, mice injected with PBS, PBS/DMSO, or left untreated (baseline) at the time point of analysis are referred to as control animals throughout the manuscript.

\section{Depletion of granulocytes}

To deplete granulocytes in vivo the anti-mouse Ly6G/Ly6C antibody (Gr1, clone RB6-8C5, BioXCell) was administered i.v. (200 $\mu \mathrm{g} /$ mouse) 36 hours prior to the mobilization treatment. Control mice were treated with an isotype control antibody (clone 2A3, BioXCell). Efficient depletion of peripheral blood granulocytes was confirmed using differential blood count as well as flow cytometric analysis.

\section{Induction of diabetes}

Diabetes was induced in 8-week-old BALB/cJ mice by a single i.p. injection of $200 \mathrm{mg} / \mathrm{kg}$ streptozotocin (SZT, EMD Millipore) dissolved in citrate buffer ( $\mathrm{pH}$ 4.7-5.3, Sigma-Aldrich). Blood glucose levels were measured with a portable blood glucose meter (Glucocard Vital, ARKRAY USA Inc.). Animals with glucose values higher than $300 \mathrm{mg} / \mathrm{dl}$ were used for mobilization experiments 2-3 weeks after SZT injection.

\section{Transplantation}

Serial competitive transplantation. Lethally irradiated CD $45.2^{+} \mathrm{BALB} / \mathrm{cJ}$ hosts received i.v. transplants consisting of $2.5 \times 10^{5} \mathrm{CD} 45.2^{+} \mathrm{BM}$ cells and a small volume $(10 \mu \mathrm{l})$ of differentially mobilized blood (BALB/cJ CD 45.1 $1^{+}$G-CSF for 5 days vs. tGro- $\beta$ vs. VLA4 antagonist firategrast or CWHM-823 vs. VLA4 antagonist plus tGro- $\beta$ ). Twenty weeks after the transplantation, $\mathrm{PB}$ composition of the recipients was analyzed for the presence of blood graft-derived hematopoiesis (i.e. percent contribution of blood donor CD $45.1^{+}$cells within the $\mathrm{CD}^{4} 5^{+} \mathrm{CD} 3^{-}$compartment). Following PB analysis, BM cells of the primary recipients were isolated and pooled based on WBC counts at equal proportion per recipient per group (G-CSF and both VLA4 antagonist plus tGro- $\beta$ groups). Primary recipient BM cell mixture $\left(2.5 \times 10^{6}\right.$ per mouse $)$ was injected into lethally irradiated secondary recipients $\left(\mathrm{CD} 45.2^{+}\right)$. PB analysis of secondary hosts was performed 18 weeks after the transplantation. Similarly, CD $45.1^{+}$percentage within the $C D 45^{+} \mathrm{CD}^{-}$fraction was determined.

Generation of transplantation chimeras for mobilization. To generate hematopoietic specific CXCR2-KO mice, $1 \times 10^{6} \mathrm{BM}$ cells from CXCR2$\mathrm{KO}$ animals (CD45.2 ${ }^{+}$) were transplanted into lethally irradiated WT syngeneic BALB/cJ CD 45.1 $1^{+}$recipients. Control group of CD $45.1^{+}$hosts received WT CD $45.2^{+}$BM cells. Conversely, stromal-specific knockouts were generated by transplanting lethally irradiated CXCR2-KO animals with $1 \times 10^{6} \mathrm{CD} 45.1^{+}$WT BM cells. In the corresponding control group, WT CD $45.2^{+}$hosts received CD $45.1^{+}$BM grafts.

For EC-specific CXCR2 ablation, lethally irradiated CXCR2 $2^{\mathrm{f} / \mathrm{fl}}$ Cdh5Cre ${ }^{+}$hosts (C57BL/6 background, CD $45.2^{+}$) were reconstituted with syngeneic WT CD $45.1^{+} \mathrm{BM}\left(3 \times 10^{6}\right.$ cells per recipient). In the control group CXCR2 $2^{\mathrm{f} / \mathrm{WT}} \mathrm{Cre}^{+}$and $\mathrm{CXCR} 2^{\mathrm{WT} / \mathrm{WT}} \mathrm{Cre}^{+}$mice were used as recipients.

Mobilization studies in chimeric animals were started 8-12 weeks after the transplantation. Lack of contribution of recipient endogenous hematopoiesis was verified by flow cytometry in each recipient.

\section{Fluorescence activated cell analysis and sorting (FACS)}

Cell labeling was performed according to standard protocols using established marker panels for identification of different subsets in mouse hematopoietic tissues as previously described $(10,12)$. Lineage-negative and c-Kit-positive $\left(\mathrm{Lin}^{-} \mathrm{kit}^{+}\right)$or lineage-negative, Sca-1-positive and c-Kit-positive cells (LSK cells) were an estimate for HSPCs, whereas HSCs were identified as LSK SLAM (LSK CD150 ${ }^{+}$ CD48-) cells. Antibodies used in this study are listed in Supplemental Table 6. Sample acquisition was performed on Gallios (Beckman Coulter Life Sciences) and BD LSRFortessa (BD Biosciences) flow cytometers or BD FACS Aria II cell sorter (BD Biosciences). Data were further analyzed using FlowJo software (Tree Star) or FACSDiva software (BD Biosciences). Cell isolation by flow sorting was performed on BD FACS Aria II. Cell-cycle analysis was performed as previously described $(10,12)$. 


\section{Colony-forming unit assay}

Cells were incubated in duplicate in commercially available growth factor supplemented with methylcellulose medium for mouse CFU-Cs (Stem Cell Technologies or R\&D Systems) as described (10, 12). CFU-Cs (BFU-E, CFU-GM, and CFU-GEMM) were enumerated after 6-8 days of culture.

\section{Quantitative real-time PCR}

For analysis of gene expression in cell populations (LSK, LSK SLAM, and different mature cell fractions) sorted from the BM RNA was isolated using RNA XS column kit (Machery-Nagel). Ambion Turbo DNA-free Kit (Thermo Fisher Scientific) was then used to remove genomic DNA followed by reverse transcription of the RNA using the QuantiTect Reverse Transcription Kit (Qiagen). qRT-PCR (Cxcr2 and Gapdh) was performed using TaqMan Master Mix, probes and primers (Applied Biosystems) listed in Supplemental Table 7.

\section{Microarray analysis}

RNA from LSK cells sorted from untreated BM or PB of differentially mobilized (G-CSF [5 days], AMD3100, or CWHM-823 plus Gro- $\beta$; single injection) mice was prepared using the RNA XS column kit (Macherey-Nagel) and hybridized to the Mouse Gene Expression v2 $4 \times 44 \mathrm{~K}$ microarray (Agilent Technologies). Normalization and quality assessment of expression data were performed using Partek Genomic Suite (Partek Inc.). Thus, $\log _{2}$ scale-transformed and filtered expression data were used for PCA plot generation. Hierarchical clustering was performed using the top 1000 differentially (significance) regulated genes. All original microarray data were deposited in the NCBI's Gene Expression Omnibus database (GSE123505) and expression data can be accessed at https://www. ncbi.nlm.nih.gov/geo/query/acc.cgi?acc=GSE123505.

\section{Pharmacokinetics}

PB was drawn from the facial vein without anesthesia and collected into lithium heparin anticoagulated tubes (Sarstedt AG \& Co). If not processed immediately, samples were stored on ice (approximately 1 hour). After centrifugation ( 25 minutes, $800-1500 g, 4^{\circ} \mathrm{C}$ ) plasma supernatant was carefully removed, frozen, and stored at $-80^{\circ} \mathrm{C}$ until just before analysis. Plasma samples or standards prepared in plasma matrix $(50 \mu \mathrm{l})$ were added to a 96-well plate. To each well, $200 \mu \mathrm{l}$ cold acetonitrile containing $100 \mathrm{ng} / \mathrm{ml}$ extraction internal standard enalapril was added. The plate was vortexed for 5 minutes, and then centrifuged at $1600 \mathrm{~g}$ for $5 \mathrm{~min}$ utes at $4^{\circ} \mathrm{C}$. The supernatant $(200 \mu \mathrm{l})$ was transferred to a second 96-well plate, evaporated to dryness under nitrogen, reconstituted with $100 \mu \mathrm{l}$ of $0.1 \% \mathrm{vol} / \mathrm{vol}$ formic acid in 9:1 water/acetonitrile, vortexed for 5 minutes, and the samples were analyzed by LC/ MS. CWHM-823 concentrations were determined on a Sciex API-4000 LC/MS system (SCIEX) in positive electrospray mode. Analytes were eluted from an Armor C18 reverse phase column $(2.1 \times 30 \mathrm{~mm}, 5 \mu \mathrm{m})$ using a $0.1 \%$ formic acid mobile phase system with aqueous to acetonitrile gradient over 3.7 minutes at a flow rate of $0.35 \mathrm{ml} / \mathrm{min}$. Peak areas for the mass transition of $\mathrm{m} / \mathrm{z} 518>472$ for CWHM-823 and m/z $376>91$ for enalapril (IS) were integrated using Analyst 1.5.1 software. Peak area ratios of CWHM-823 area/ enalapril area were plotted against standard concentrations with a $1 / \mathrm{x}$-weighted linear regression.

\section{Water solubility assay}

For each VLA4 antagonist tested, 1-2 mg solid compound was placed in an Eppendorf tube with $1 \mathrm{ml}$ equilibrium solubility buffer (ESB, 50 $\mathrm{mM}$ citric acid, $50 \mathrm{mM} \mathrm{K}_{2} \mathrm{HPO}_{4}, 50 \mathrm{mM}$ Ammediol, and $50 \mathrm{mM} \mathrm{KCl}$, $\mathrm{pH} 7$ ) and incubated in a Thermomixer for 48 hours at $25^{\circ} \mathrm{C}$ at $500 \mathrm{rpm}$. After 24 hours, tubes were centrifuged at $3000 \mathrm{~g}$ for 5 minutes, and an aliquot of the supernatant was removed and diluted into linear standard range of the standard curve for the LCMS method. Sample dilutions and standards were made in 75:25 ESB/acetonitrile. Firategrast, BIO5192, CWHM-823, CWHM-824, CWHM-825, and CWHM-842 concentrations were determined on a Sciex API-4000 LC/MS system (SCIEX) in positive electrospray mode. Analytes were eluted from an Armor C18 reverse phase column $(2.1 \times 30 \mathrm{~mm}, 5 \mu \mathrm{m})$ using a $0.1 \%$ formic acid mobile phase system with aqueous to acetonitrile gradient over 3.7 minutes at a flow rate of $0.35 \mathrm{ml} / \mathrm{min}$. Peak areas for the mass transition of $\mathrm{m} / \mathrm{z} 500.3>454.2$ for firategrast, $\mathrm{m} / \mathrm{z} 817.2>394.0$ for BIO5192, m/z $505.0>176.0$ for CWHM-822, m/z $518.0>472.2$ for CWHM-823, m/z 621.2 > 575.0 for CWHM-824, m/z 474.6 > 192.2 for CWHM-825, and m/z 523.4 > 390.2 for CWHM-842 were integrated using Analyst 1.5.1 software (SCIEX). Peak areas were plotted against standard concentrations with a $1 / \mathrm{x}$-weighted linear regression.

\section{VCAM1 binding assay}

G2 ALL cells $\left(1.5 \times 10^{5}\right.$ cells per sample, The Hospital for Sick Children) were incubated with increasing concentrations of the different VLA4 antagonists for 30 minutes at room temperature (RT). Subsequently, human recombinant VCAM1/Fc chimera protein (R\&D Systems) was added to the samples at a final concentration of $10 \mu \mathrm{g} / \mathrm{ml}$. After a second 30-minute incubation step at RT, cells were washed twice with HBSS/0.1\% BSA buffer and secondary antibody (PE-labeled donkey anti-human IgG, catalog 709-116-098, Jackson Immunoresearch Laboratories) added at 1:100 dilution. Control samples were stained with PE-labeled donkey IgG (catalog 017-110-006, Jackson Immunoresearch Laboratories, 1:100 dilution). Secondary antibody staining was performed at RT for 30 minutes. Following addition of 7-AAD for 5 minutes at RT, cells were washed twice with HBSS/BSA buffer and analyzed by flow cytometry.

\section{Integrin binding assay}

The potency of compounds in blocking ligand binding to integrins $\mathrm{a} 4 \mathrm{~b} 1$ and $\mathrm{a} 4 \mathrm{~b} 7$ was determined by modification of our previously described methods (71). Briefly, purified human VCAM1 (R\&D Systems) diluted to $5 \mu \mathrm{g} / \mathrm{ml}$ in $\mathrm{TBS}^{+}$buffer ( $25 \mathrm{mM}$ Tris pH 7.4, 137 $\mathrm{mM} \mathrm{NaCl}, 2.7 \mathrm{mM} \mathrm{KCl}, 1 \mathrm{mM} \mathrm{CaCl}, 1 \mathrm{mM} \mathrm{MgCl}, 1 \mathrm{mM} \mathrm{MnCl}_{2}$ ) was added to wells of a 96-well transparent microtiter plate and incubated overnight at $4^{\circ} \mathrm{C}$. Wells were washed 3 times with $\mathrm{TBS}^{+}$and blocking buffer ( $\mathrm{TBS}^{+}$with $1 \%$ bovine serum albumin), the plate was incubated for 1 hour at $37^{\circ} \mathrm{C}$, and then washed 3 times with $\mathrm{TBS}^{+}$ buffer. Recombinant human integrin ITGA4/ITGB1 ( $\alpha 4 \beta 1$; VLA4) or ITGA4/ITGB7 ( $\alpha 4 \beta 7$ ) (R\&D Systems) was diluted to $1 \mu \mathrm{g} / \mathrm{ml}$ in $\mathrm{TBS}^{+} / 0.1 \%$ bovine serum albumin. Test compounds were diluted into the integrin solution and added to the washed ligand-coated plate according to a standard template with each sample repeated in triplicate. After incubation for 2 hours at room temperature, the plate was washed 3 times with $150 \mu \mathrm{TBS}^{+}$buffer. To each well, biotinylated anti- $\beta 1$ (catalog BAF1778, R\&D Systems) or anti- $\alpha 4$ antibody (clone $7.2 \mathrm{R}, \mathrm{R} \& \mathrm{D}$ Systems) at $1 \mu \mathrm{g} / \mathrm{ml}$ in $\mathrm{TBS}^{+} / 0.1 \%$ BSA 
was added and the plate covered and incubated for 1 hour at RT. After washing the plate 3 times with $\mathrm{TBS}^{+}$buffer, streptavidinconjugated horseradish peroxidase (R\&D Systems) diluted in $\mathrm{TBS}^{+}$ blocking buffer was added to the wells and the plate incubated for 20 minutes at room temperature. The plate was washed 3 times with $\mathrm{TBS}^{+}$buffer followed by addition of $50 \mu \mathrm{l}$ TMB substrate (MilliporeSigma). After incubation for 20 minutes at RT, plates were read by colorimetric detection at $650 \mathrm{~nm}$ wavelength using a Tecan Safire II plate reader. Concentration-response curves were constructed by nonlinear regression (best fit) analysis, and $\mathrm{IC}_{50}$ values were calculated for each compound.

To evaluate the broader selectivity of test compounds, potency against a nontargeted beta- 1 subunit-containing integrin, ITGA2/ ITGB1 ( $\alpha 2 \beta 1)$, was measured as previously described (71). Assessment of compound potency against 2 selected members of the RGD-binding integrin family, ITGA5/ITGB1 ( $\alpha 5 \beta 1)$ and ITGAV/ ITGB3 ( $\alpha$ v 33 ), was determined by a similar method in which binding of the purified human integrins (R\&D Systems) was assessed to plates coated with their respective purified ligands, human fibronectin ( $2 \mu \mathrm{g} / \mathrm{ml}, \mathrm{R} \& \mathrm{D}$ Systems) and human vitronectin $(1 \mu \mathrm{g} /$ $\mathrm{ml} ; \mathrm{R} \& \mathrm{D}$ Systems). Biotinylated anti-ITGAV ( $\alpha \mathrm{v})$ or anti-ITGA5 ( $\alpha 5 ;$ R\&D Systems) antibodies were used to detect the stably bound integrins in conjunction with streptavidin-conjugated horseradish peroxidase as described above. The $\alpha v \beta 3$ and $\alpha 5 \beta 1$ assays were validated by inclusion of a known potent inhibitor of these integrins, CWHM-12 (71), while the $\alpha 2 \beta 1$ assay was validated by inclusion of another previously described inhibitor, compound 8 (data not shown) (72).

\section{Specificity screening}

tGro- $\beta$ was sent out to DiscoverX (DiscoverX Corporation) for blinded profiling against the gpcrMAX panel ( $148 \mathrm{G}$ protein-coupled receptors screened in both, agonist and antagonist mode) using the PathHunter beta-arrestin enzyme fragmentation (EFC) technology.

\section{Statistics}

Data are mean \pm SEM unless indicated otherwise. Linear mixed models were used to analyze experiments with data repeatedly measured from the same mice, while analysis of variance (ANOVA) was used for data from independent samples. A logarithm transformation was performed as necessary to better satisfy the normality and homoscedasticity assumptions (see Figure legends for details). Ad-hoc multiple comparisons were also used for between-group differences of interest. The resultant $P$ values were adjusted by Holm's step-down Bonferroni's adjustment. Compared with the widely used Bonferroni's adjustment, a step-down method is more powerful (smaller adjusted $P$ values) while maintaining strong control of the familywise error rate. All analyses were 2 -sided and significance was set at a $P$ value of 0.05. The statistical analyses were performed using SAS 9.4 (SAS Institutes).

\section{Study approval}

All animals were housed at the Washington University Medical School vivarium under SPF conditions with autoclaved chow and water ad libitum. All experiments were performed in accordance with the guidelines of the Washington University Animal Studies Committee, approved by the IACUC in agreement with AAALAC guidelines.

\section{Author contributions}

DK, MPR, and JFD conceived of the study. DK, MPR, JR, SC, LG, EC, DC, MOE, MH, JZ, GA, HC, EW, WY, LGE, RFH, SDA, MJM, MJP, DWG, PGR, and DMM performed experiments. DK, MPR, and DC analyzed data. WY assisted with microarray data processing and analysis. FG assisted with the statistical analysis of the data. DK, MPR, AT, HBB, DCL, and JFD supervised research. DK wrote the manuscript. All authors discussed, commented on, and approved the final version of the manuscript.

\section{Acknowledgments}

DK is a past scholar of the German Academic Exchange Service (postdoctoral fellowship ID: 57054578, 2014-2016). MPR is supported by the National Institutes of Health, National Cancer Institute (R50 CA211466), an Alvin J. Siteman Cancer Center, Siteman Investment Program award that is supported by The Foundation for Barnes-Jewish Hospital Cancer Frontier Fund, the Barnard Trust, and a National Cancer Institute Cancer Center support grant (P30 CA091842). HC was supported by the American Society of Hematology and the Edward P. Evans Foundation. HB is a member of LOEWE Cell and Gene Therapy Frankfurt faculty, funded by the Hessian Ministry of Higher Education, Research and the Arts (III L 4- 518/17.004, 2010-2018). JFD is supported by grants from the National Institutes of Health, National Cancer Institute (U54 CA199092, R01 CA152329, and R35 CA210084). We thank the Genome Technology Access Center in the Department of Genetics at Washington University School of Medicine for help with genomic analysis. The Genome Technology Access Center is partially supported by NCI Cancer Center support grant P30 CA91842 to the Siteman Cancer Center and by ICTS/CTSA grant UL1RR024992 from the National Center for Research Resources (NCRR), a component of NIH, and NIH Roadmap for Medical Research. This publication is solely the responsibility of the authors and does not necessarily represent the official view of NCRR or NIH. We thank the Alvin J. Siteman Cancer Center at Washington University School of Medicine and Barnes-Jewish Hospital in St. Louis, Missouri, for the use of the Siteman Flow Cytometry Core. The Siteman Cancer Center is supported in part by an NCI Cancer Center support grant P30 CA91842.

Address correspondence to: John F. DiPersio, Division of Oncology, Washington University School of Medicine, 660 South Euclid Avenue, Campus Box 8007, St. Louis, Missouri 63110, USA. Phone: 314.454.8491; Email: jdipersi@wustl.edu.

RFH and SDA's present address is: Confluence Discovery Technologies, St. Louis, Missouri, USA.

MJM's present address is: Department of Chemistry, Saint Louis University, St. Louis, Missouri, USA.

MJP's present address is: HTS Robotics Core, Washington University School of Medicine, St. Louis, Missouri, USA.

DWG's present address is: Department of Molecular Microbiology \& Immunology, Saint Louis University School of Medicine, St. Louis, Missouri, USA. 
1. Gao X, Xu C, Asada N, Frenette PS. The hematopoietic stem cell niche: from embryo to adult. Development. 2018;145(2):dev139691.

2. Frisch BJ. The hematopoietic stem cell niche: what's so special about bone? Bone. 2019;119:8-12.

3. Molineux G, Pojda Z, Hampson IN, Lord BI, Dexter TM. Transplantation potential of peripheral blood stem cells induced by granulocyte colony-stimulating factor. Blood.1990;76(10):2153-2158.

4. Bonig H, Papayannopoulou T. Hematopoietic stem cell mobilization: updated conceptual renditions. Leukemia. 2013;27(1):24-31.

5. Domingues MJ, Nilsson SK, Cao B. New agents in HSC mobilization. Int J Hematol. 2017;105(2):141-152.

6. Christopher MJ, Liu F, Hilton MJ, Long F, Link DC. Suppression of CXCL12 production by bone marrow osteoblasts is a common and critical pathway for cytokine-induced mobilization. Blood. 2009;114(7):1331-1339.

7. Rettig MP, Ansstas G, DiPersio JF. Mobilization of hematopoietic stem and progenitor cells using inhibitors of CXCR4 and VLA-4. Leukemia. 2012;26(1):34-53.

8. Broxmeyer HE, et al. Rapid mobilization of murine and human hematopoietic stem and progenitor cells with AMD3100, a CXCR4 antagonist. J Exp Med. 2005;201(8):1307-1318.

9. Bonig H, Chudziak D, Priestley G, Papayannopoulou T. Insights into the biology of mobilized hematopoietic stem/progenitor cells through innovative treatment schedules of the CXCR4 antagonist AMD3100. Exp Hematol. 2009;37(3):402-15.e1.

10. Karpova D, et al. The novel CXCR4 antagonist POL5551 mobilizes hematopoietic stem and progenitor cells with greater efficiency than Plerixafor. Leukemia. 2013;27(12):2322-2331.

11. Karpova D, Bonig H. Concise review: CXCR4/ CXCL12 signaling in immature hematopoiesis-lessons from pharmacological and genetic models. Stem Cells. 2015;33(8):2391-2399.

12. Karpova D, et al. Continuous blockade of CXCR4 results in dramatic mobilization and expansion of hematopoietic stem and progenitor cells. Blood. 2017;129(21):2939-2949.

13. Abraham M, et al. Enhanced unique pattern of hematopoietic cell mobilization induced by the CXCR4 antagonist 4F-benzoyl-TN14003. Stem Cells. 2007;25(9):2158-2166.

14. Bonig H, Wundes A, Chang KH, Lucas S, Papayannopoulou T. Increased numbers of circulating hematopoietic stem/progenitor cells are chronically maintained in patients treated with the CD49d blocking antibody natalizumab. Blood. 2008;111(7):3439-3441.

15. Moepps B. CXCR1 and CXCR2 and Ligands. In: Parnham MJ, ed. Compendium of Inflammatory Diseases. Basel, Switzerland: Springer International Publishing; 2016:394-404.

16. Laterveer L, Lindley IJ, Hamilton MS, Willemze R, Fibbe WE. Interleukin-8 induces rapid mobilization of hematopoietic stem cells with radioprotective capacity and long-term myelolymphoid repopulating ability. Blood. 1995;85(8):2269-2275.

17. Pelus LM, Fukuda S. Peripheral blood stem cell mobilization: the CXCR2 ligand GRObeta rapidly mobilizes hematopoietic stem cells with enhanced engraftment properties. Exp Hematol. 2006;34(8):1010-1020.

18. Fibbe WE, et al. Biology of IL-8-induced stem cell mobilization. Ann NY Acad Sci. 1999;872:71-82.

19. Pruijt JF, van Kooyk Y, Figdor CG, Lindley IJ, Willemze R, Fibbe WE. Anti-LFA-1 blocking antibodies prevent mobilization of hematopoietic progenitor cells induced by interleukin-8. Blood. 1998;91(11):4099-4105.

20. Hipkin RW, et al. Cloning and pharmacological characterization of CXCR1 and CXCR2 from Macaca fascicularis. J Pharmacol Exp Ther. 2004;310(1):291-300.

21. Karpova D, et al. Mobilization of hematopoietic stem cells with the novel CXCR4 antagonist POL6326 (balixafortide) in healthy volunteersresults of a dose escalation trial. J Transl Med. 2017;15(1):2.

22. Schroeder MA, et al. Mobilization of allogeneic peripheral blood stem cell donors with intravenous plerixafor mobilizes a unique graft. Blood. 2017;129(19):2680-2692.

23. Wei Q, Frenette PS. Niches for hematopoietic stem cells and their progeny. Immunity. 2018;48(4):632-648.

24. Miller DH, et al. Firategrast for relapsing remitting multiple sclerosis: a phase 2, randomised, double-blind, placebo-controlled trial. Lancet Neurol. 2012;11(2):131-139.

25. Kim AG, et al. Enhanced in utero allogeneic engraftment in mice after mobilizing fetal HSCs by $\alpha 4 \beta 1 / 7$ inhibition. Blood. 2016;128(20):2457-2461.

26. King AG, et al. Rapid mobilization of murine hematopoietic stem cells with enhanced engraftment properties and evaluation of hematopoietic progenitor cell mobilization in rhesus monkeys by a single injection of SB-251353, a specific truncated form of the human CXC chemokine GRObeta. Blood. 2001;97(6):1534-1542.

27. Fukuda S, Bian H, King AG, Pelus LM. The chemokine GRObeta mobilizes early hematopoietic stem cells characterized by enhanced homing and engraftment. Blood. 2007;110(3):860-869.

28. Ramirez P, et al. BIO5192, a small molecule inhibitor of VLA-4, mobilizes hematopoietic stem and progenitor cells. Blood. 2009;114(7):1340-1343.

29. Duplantier AJ, et al. Isoxazolyl, oxazolyl, and thiazolylpropionic acid derivatives as potent alpha(4)beta(1) integrin antagonists. Bioorg Med Chem Lett. 2001;11(19):2593-2596.

30. Hagmann WK, et al. The discovery of sulfonylated dipeptides as potent VLA-4 antagonists. Bioorg Med Chem Lett. 2001;11(20):2709-2713.

31. Kamenecka TM, et al. N-aryl-prolyl-dipeptides as potent antagonists of VLA-4. Bioorg Med Chem Lett. 2002;12(16):2205-2208.

32. Kudlacz E, et al. Pulmonary eosinophilia in a murine model of allergic inflammation is attenuated by small molecule alpha4beta1 antagonists. J Pharmacol Exp Ther. 2002;301(2):747-752.

33. Li B, et al. N-(arylacetyl)-biphenylalanines as potent VLA-4 antagonists. Bioorg Med Chem Lett. 2002;12(16):2141-2144.

34. Sircar I, et al. Synthesis and SAR of N-benzoylL-biphenylalanine derivatives: discovery of TR-14035, a dual alpha(4)beta(7)/alpha(4) beta(1) integrin antagonist. Bioorg Med Chem. 2002;10(6):2051-2066.

35. DiPersio JF. Diabetic stem-cell “mobilopathy". N Engl JMed. 2011;365(26):2536-2538.

36. Ferraro F, et al. Diabetes impairs hematopoietic stem cell mobilization by altering niche function. Sci Transl Med.2011;3(104):104ra101.

37. Land RH, et al. The orphan nuclear receptor NR4A1 specifies a distinct subpopulation of quiescent myeloid-biased long-term HSCs. Stem Cells. 2015;33(1):278-288.

38. Reutershan J, et al. Critical role of endothelial CXCR2 in LPS-induced neutrophil migration into the lung. J Clin Invest. 2006;116(3):695-702

39. Hale SJ, et al. CXCR2 modulates bone marrow vascular repair and haematopoietic recovery post-transplant. $\mathrm{Br} \mathrm{J} \mathrm{Haematol}$. 2015;169(4):552-564.

40. Zhang J, Link DC. Targeting of mesenchymal stromal cells by Cre-recombinase transgenes commonly used to target osteoblast lineage cells. J Bone Miner Res. 2016;31(11):2001-2007.

41. Greenbaum A, et al. CXCL12 in early mesenchymal progenitors is required for haematopoietic stem-cell maintenance. Nature. 2013;495(7440):227-230.

42. Lefort CT, Ley K. Neutrophil arrest by LFA-1 activation. Front Immunol. 2012;3:157.

43. Weber C, Springer TA. Neutrophil accumulation on activated, surface-adherent platelets in flow is mediated by interaction of Mac- 1 with fibrinogen bound to alphaIIbbeta3 and stimulated by platelet-activating factor. JClin Invest. 1997;100(8):2085-2093.

44. Seo SM, McIntire LV, Smith CW. Effects of IL-8, Gro-alpha, and LTB(4) on the adhesive kinetics of LFA-1 and Mac-1 on human neutrophils. Am J Physiol, Cell Physiol. 2001;281(5):C1568-C1578.

45. Grönholm M, et al. LFA-1 integrin antibodies inhibit leukocyte $\alpha 4 \beta 1$-mediated adhesion by intracellular signaling. Blood. 2016;128(9):1270-1281.

46. Magazin M, Vita N, Cavrois E, Lefort S, Guillemot JC, Ferrara P. The biological activities of gro beta and IL-8 on human neutrophils are overlapping but not identical. Eur Cytokine Netw. 1992;3(5):461-467.

47. Velders GA, et al. Enhancement of G-CSFinduced stem cell mobilization by antibodies against the beta 2 integrins LFA- 1 and Mac- 1 . Blood. 2002;100(1):327-333.

48. Hidalgo A, Peired AJ, Weiss LA, Katayama Y, Frenette PS. The integrin alphaMbeta2 anchors hematopoietic progenitors in the bone marrow during enforced mobilization. Blood. 2004;104(4):993-1001.

49. Fibbe WE, Pruijt JF, van Kooyk Y, Figdor CG Opdenakker G, Willemze R. The role of metalloproteinases and adhesion molecules in interleukin-8-induced stem-cell mobilization. Semin Hematol. 2000;37(1 Suppl 2):19-24.

50. Pruijt JF, et al. Prevention of interleukin-8induced mobilization of hematopoietic progenitor cells in rhesus monkeys by inhibitory antibodies against the metalloproteinase gelatinase B (MMP-9). Proc Natl Acad Sci US A. 1999;96(19):10863-10868.

51. Pelus LM, Bian H, King AG, Fukuda S. Neutrophil- 
derived MMP-9 mediates synergistic mobilization of hematopoietic stem and progenitor cells by the combination of G-CSF and the chemokines GRObeta/CXCL2 and GRObetaT/CXCL2delta4. Blood. 2004;103(1):110-119.

52. Hoggatt J, et al. Rapid mobilization reveals a highly engraftable hematopoietic stem cell. Cell. 2018;172(1-2):191-204.e10.

53. Morrison SJ, Wright DE, Weissman IL. Cyclophosphamide/granulocyte colony-stimulating factor induces hematopoietic stem cells to proliferate prior to mobilization. Proc Natl Acad Sci U S A. 1997;94(5):1908-1913.

54. Winkler IG, Wiercinska E, Barbier V, Nowlan B, Bonig H, Levesque JP. Mobilization of hematopoietic stem cells with highest self-renewal by G-CSF precedes clonogenic cell mobilization peak. Exp Hematol. 2016;44(4):303-14.e1.

55. Liu F, Poursine-Laurent J, Link DC. Expression of the G-CSF receptor on hematopoietic progenitor cells is not required for their mobilization by G-CSF. Blood. 2000;95(10):3025-3031.

56. Lévesque JP, Helwani FM, Winkler IG. The endosteal 'osteoblastic' niche and its role in hematopoietic stem cell homing and mobilization. Leukemia. 2010;24(12):1979-1992.

57. Grassinger J, Williams B, Olsen GH, Haylock DN, Nilsson SK. Granulocyte colony stimulating factor expands hematopoietic stem cells within the central but not endosteal bone marrow region. Cytokine. 2012;58(2):218-225.
58. Fujisaki J, et al. In vivo imaging of Treg cells providing immune privilege to the haematopoietic stem-cell niche. Nature. 2011;474(7350):216-219.

59. Bowers E, Slaughter A, Frenette PS, Kuick R, Pello OM, Lucas D. Granulocyte-derived TNF $\alpha$ promotes vascular and hematopoietic regeneration in the bone marrow. Nat Med. 2018;24(1):95-102.

60. Pruijt JF, et al. Neutrophils are indispensable for hematopoietic stem cell mobilization induced by interleukin-8 in mice. Proc Natl Acad Sci U S A. 2002;99(9):6228-6233.

61. Blaser BW, et al. CXCR1 remodels the vascular niche to promote hematopoietic stem and progenitor cell engraftment. J Exp Med. 2017;214(4):1011-1027.

62. Pruijt JF, van Kooyk Y, Figdor CG, Willemze R, Fibbe WE. Murine hematopoietic progenitor cells with colony-forming or radioprotective capacity lack expression of the beta 2-integrin LFA-1. Blood.1999;93(1):107-112.

63. Papayannopoulou T. Mechanisms of stem-/ progenitor-cell mobilization: the anti-VLA-4 paradigm. Semin Hematol. 2000;37(1 Suppl 2):11-18.

64. Schraufstatter IU, Chung J, Burger M. IL-8 activates endothelial cell CXCR1 and CXCR2 through Rho and Rac signaling pathways. Am J Physiol Lung Cell Mol Physiol. 2001;280(6):L1094-L1103.

65. Lei X, Hossain M, Qadri SM, Liu L. Different microvascular permeability responses elicited by the CXC chemokines MIP-2 and KC during leukocyte recruitment: role of LSP1. Biochem Biophys Res Commun. 2012;423(3):484-489.

66. Gavard J, et al. A role for a CXCR2/phosphatidylinositol 3-kinase gamma signaling axis in acute and chronic vascular permeability. Mol Cell Biol. 2009;29(9):2469-2480.

67. Dwyer J, et al. Glioblastoma cell-secreted interleukin-8 induces brain endothelial cell permeability via CXCR2. PLOS ONE. 2012;7(9):e45562.

68. Borkowska S, et al. Novel evidence that crosstalk between the complement, coagulation and fibrinolysis proteolytic cascades is involved in mobilization of hematopoietic stem/progenitor cells (HSPCs). Leukemia. 2014;28(11):2148-2154.

69. Ratajczak MZ, Adamiak M, Kucia M, Tse W, Ratajczak J, Wiktor-Jedrzejczak W. The emerging link between the complement cascade and purinergic signaling in stress hematopoiesis. Front Immunol. 2018;9:1295.

70. Ratajczak MZ, Adamiak M. Membrane lipid rafts, master regulators of hematopoietic stem cell retention in bone marrow and their trafficking. Leukemia. 2015;29(7):1452-1457.

71. Henderson NC, et al. Targeting of av integrin identifies a core molecular pathway that regulates fibrosis in several organs. Nat Med.2013;19(12):1617-1624.

72. Wilkinson AL, Barrett JW, Slack RJ. Pharmacological characterisation of a tool $\alpha v \beta 1$ integrin small molecule RGD-mimetic inhibitor. Eur J Pharmacol. 2019;842:239-247. 